Check for updates

Cite this: RSC Adv., 2017, 7, 25285

Received 8th February 2017

Accepted 17th April 2017

DOI: $10.1039 / \mathrm{c} 7 \mathrm{ra01609c}$

rsc.li/rsc-advances

\section{Bicyclic lactones and racemic mixtures of dimeric styrylpyrones from the leaves of Miliusa velutinat}

\author{
N. Wongsa, ${ }^{\text {ab }}$ K. Kanokmedhakul, (D) *a J. Boonmak, (D) S. Youngme ${ }^{c}$ \\ and S. Kanokmedhakul ${ }^{\mathrm{a}}$
}

A unique class of eight bicyclic lactones with a $\mathrm{C}_{18}$ carbon architecture, named velutinones $\mathrm{A}-\mathrm{H}(1-8)$, three new dimeric styrylpyrones, velutinindimers A-C (9-11), five known compounds, the kawapyrone, yangonin (12), three flavonoids (13-15), and an acetogenin, cananginone $H(16)$ were isolated from the leaves of Miliusa velutina. The absolute configurations of 2 and 5 were assigned by Mosher's method, whereas $E C D$, optical rotations, and $\mathrm{X}$-ray crystallographic analysis indicated the racemic nature of compounds 10 and 11 . Compounds 2-4 and 7-11 showed antimalarial activity with $I C_{50}$ values in the range of 5.4$10.0 \mu \mathrm{M}$. Moreover, 1-4 and 6-8 displayed cytotoxicity against the KB, MCF7, and $\mathrm{NCl}-\mathrm{H} 187$ cancer cell lines and Vero cell lines with $\mathrm{IC}_{50}$ values in the range of $4.0-24.1 \mu \mathrm{M}$.

\section{Introduction}

Miliusa velutina (Dunal) Hook. f. \& Thomson belongs to the family Annonaceae. This plant is found widely in Thailand with local names "Khang hua mu" or "Kong kang". A water decoction of the wood is used traditionally as a tonic and an aphrodisiac. ${ }^{1}$ The genus Miliusa comprises ca. 50 species distributed from India, South East Asia, to Australia. At least 19 species of Miliusa, have been found in Thailand. ${ }^{2,3}$ Eight of the Miliusa genera growing worldwide have been investigated for their phytochemistry and biological activities. ${ }^{4-19}$ Among these species, a Thai medicinal plant, M. velutina, has been shown to contain the acetogenin, goniothalamusin, ${ }^{17}$ an aporphine alkaloid, (+)-isocorydine $\alpha-N$ oxide, ${ }^{18}$ and four alkaloids, reticuline, liriodenine, norcorydine, and isocorydine. ${ }^{19}$ Recently, the isolation and characterization of the linear acetogenins, cananginones A-I from the stem bark of $M$. velutina were reported..$^{20,21}$ In a continued investigation of this plant, the crude $n$-hexane and EtOAc extracts from the leaves of this plant were found to exhibit activity towards Mycobacterium tuberculosis with 99.6 and $98.9 \%$ inhibition at a concentration of $50 \mu \mathrm{g} \mathrm{mL}{ }^{-1}$, respectively. Herein the isolation, structural identification, and bioactivities of eight new bicyclic lactones (1-8),

${ }^{a}$ Natural Products Research Unit, Department of Chemistry and Center of Excellence for Innovation in Chemistry, Faculty of Science, Khon Kaen University, Khon Kaen 40002, Thailand.E-mail: kwanjai@kku.ac.th

${ }^{b}$ Division of Chemistry, Faculty of Science, Udon Thani Rajabhat University, Udon Thani 41000, Thailand

${ }^{c}$ Materials Chemistry Research Center, Department of Chemistry and Center of Excellence for Innovation in Chemistry, Faculty of Science, Khon Kaen University, Khon Kaen 40002, Thailand

$\dagger$ Electronic supplementary information (ESI) available: 1D NMR spectra of new compounds 1-11. CCDC 1415288 and 1062125. For ESI and crystallographic data in CIF or other electronic format see DOI: 10.1039/c7ra01609c and three new cyclobutane dimers (9-11), as well as five known compounds (12-16) from the leaves of $M$. velutina are discussed (Fig. 1).

\section{Experimental section}

\subsection{General procedures}

A Gallenkamp melting point apparatus $\left(0-300{ }^{\circ} \mathrm{C}, 4{ }^{\circ} \mathrm{C} \mathrm{min}^{-1}\right.$, uncorrected) was used to measure melting points. Optical rotations were recorded on a JASCO P-1020 polarimeter and ECD spectra were recorded on a JASCO J-810 apparatus. UV spectra were recorded using an Agilent 8453 UV-visible spectrophotometer. FTIR spectra were recorded on a Bruker Tensor 27 spectrophotometer. The NMR spectra were acquired on a Varian Mercury Plus 400 spectrometer. HRESITOFMS spectra were recorded on a Micromass Q-TOF-2 mass spectrometer. Flash column chromatography (FCC) was performed on MERCK silica gel 60 (230-400 mesh) and LiChroprep ${ }^{\circledR}$ RP-18 (40-63 $\mu \mathrm{m})$. Thin layer chromatography (TLC) was performed using precoated MERCK silica gel $60 \mathrm{PF}_{254}$ and RP-18 $\mathrm{F}_{254} \mathrm{~S}$.

\subsection{Plant material}

The leaves of $M$. velutina were collected in Nam Som district, Udon Thani province, Thailand in November 2010. The identification of the plant was performed by Prof. Pranom Chantaranothai. A voucher specimen (S. Kanokmedhakul-17) was deposited at the herbarium of the Department of Biology, Faculty of Science, Khon Kaen University, Thailand. It should be noted that, in our previous report $^{20}$ on this plant, it was collected in different locations and was misidentified as Cananga latifolia because of incomplete material for species identification. It was identified based on the vegetative part (leaf and 
<smiles></smiles>

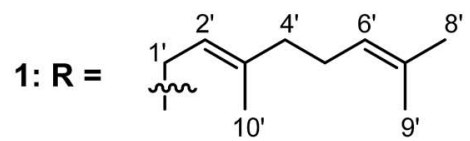

2: $\mathbf{R}=$<smiles>[R]O[C@@H](CC/C(C)=C/C(C)C#[R])C([B])=[B]</smiles>

2: $\mathrm{R}_{1}=\mathrm{H}$

2a: $\mathrm{R}_{1}=$ S-MTPA

2b: $\mathrm{R}_{1}=R-\mathrm{MTPA}$<smiles>CC(=CCN(C)C=[W])CC=CC(C)(C)O</smiles><smiles>[Z4]C#[Y]C/C=C(\C)C/C=C/C(C)(C)O</smiles><smiles>[R20]O[C@H]1C=C[C@H]2OC(=O)C[C@@]2(C/C=C(\C)CCC=C(C)C)C1</smiles>

5: $\mathrm{R}_{1}=\mathrm{H}$

5a: $\mathrm{R}_{1}=$ S-MTPA

5b: $\mathrm{R}_{1}=R$-MTPA<smiles>[R][C@]12CC(=O)O[C@H]1[C@H]1O[C@H]1C(=O)C2</smiles>

6: $\mathbf{R}=\overbrace{1}^{1}$<smiles>[R]#[W]CC=C(C)CC[C@H](O)C(=C)C</smiles><smiles>[R][N+](C)(C)CC=C(C)CCC1OC1(C)C</smiles><smiles>C=CCCCC/C=C\C#CCCCCCC[C@H]1C[C@H](CO)OC1=O</smiles><smiles>COc1ccc([C@@H]2[C@H](c3cc(OC)cc(=O)o3)[C@H](c3ccc(OC)cc3)[C@@H]2c2ccc(OC)cc2)cc1</smiles><smiles>COc1ccc(/C=C/c2cc(OC)cc(=O)o2)cc1</smiles>

12<smiles>[R]c1ccc([C@H]2CC(=O)c3c(O)cc(OC)cc3O2)cc1[R2]</smiles>

13: $\mathrm{R}_{1}=\mathrm{H}, \mathrm{R}_{2}=\mathrm{OH}$

14: $R_{1}=R_{2}=O H$<smiles>COc1cc(O)c2c(=O)c(O)c(-c3ccc(O)c(O)c3)oc2c1</smiles>

15

Fig. 1 Structures of isolated compounds 1-16.

stem). Later in 2015, plants with fruit from both locations were collected and the samples identified and confirmed as Miliusa velutina, and this has been corrected as an erratum. ${ }^{21}$ Since the work has been published for some time, to avoid any future confusion, the names of the new compounds have not been changed.

\subsection{Extraction and isolation}

The dried, milled leaves of M. velutina $(2.5 \mathrm{~kg})$ were extracted with $n$-hexane $(3 \times 10 \mathrm{~L})$ and EtOAc $(3 \times 10 \mathrm{~L})$ to give $127 \mathrm{~g}$ $(5.1 \%)$ and $93 \mathrm{~g}(3.7 \%)$ of $n$-hexane and EtOAc extracts, respectively. The $n$-hexane extract was separated using silica gel flash column chromatography (FCC), eluted with a gradient system of $n$-hexane-EtOAc $(100: 0,90: 10,85: 15,70: 30$, 
$50: 50,40: 60,20: 80,0: 100)$ and EtOAc-MeOH, EtOAc$\mathrm{MeOH}(80: 20,50: 50,30: 70,15: 85,0: 100)$ to afford 5 fractions, $\mathrm{LH}_{1}-\mathrm{LH}_{5}$. Fraction $\mathrm{LH}_{3}(48.2 \mathrm{~g})$ was chromatographed on silica gel FCC, eluting with $n$-hexane-acetone $(4: 1)$ to yield 3 subtractions, $\mathrm{LH}_{3.1}-\mathrm{LH}_{3.3}$. Subfraction $\mathrm{LH}_{3.2}$ (32.8 g) was purified by LiChroprep RP-18 column chromatography, eluted with $\mathrm{MeOH}-\mathrm{H}_{2} \mathrm{O}(4: 1)$ to give 6 fractions, $\mathrm{LH}_{3.2 .1}-\mathrm{LH}_{3.2 .6}$. Further purification of subfraction $\mathrm{LH}_{3.2 .1}(0.48 \mathrm{~g})$ by silica gel FCC, eluting with $n$-hexane-EtOAc ( $4: 1)$ gave compounds 4 (53.8 mg) and $2(23 \mathrm{mg})$ as colorless viscous liquids. Subfraction $\mathrm{LE}_{3.2 .2}$ $(23.6 \mathrm{~g})$ was separated on silica gel FCC, eluting with $n$-hexaneEtOAc (85: 15) to give $\mathbf{1}(20.3 \mathrm{~g}$ ) as a colorless viscous liquid. Subfraction $\mathrm{LH}_{3.2 .3}(1.7 \mathrm{~g})$ was chromatographed on silica gel FCC, eluting with $n$-hexane-EtOAc (85: 15) to give $1(1.59 \mathrm{~g})$ and $6(57.3 \mathrm{mg})$ as colorless viscous liquids. Subfraction $\mathrm{LH}_{3.3}(9.6 \mathrm{~g})$ was purified by silica gel FCC, eluting with $n$-hexane-acetone ( $7: 1$ ) to afford 5 subfractions, $\mathrm{LH}_{3.3 .1}-\mathrm{LH}_{3.3 .5}$. Further purification of subfraction $\mathrm{LH}_{3.3 .1}(0.28 \mathrm{~g})$ by silica gel FCC, eluted with $n$-hexane-EtOAc $(3: 1)$ gave $8(49.8 \mathrm{mg})$ as a colorless viscous liquid. Subfraction $\mathrm{LE}_{3.3 .2}(0.49 \mathrm{~g})$ was chromatographed on silica gel FCC, eluted with $n$-hexane-EtOAc $(1: 1)$ to afford $7(408.5 \mathrm{mg})$ as a colorless viscous liquid. Subfraction $\mathrm{LH}_{3.4 .4}(0.68 \mathrm{~g})$ was purified by silica gel FCC, eluting with $n$ hexane-EtOAc (75 : 35) to give an extra amount of $2(551.7 \mathrm{mg})$. Subfraction $\mathrm{LH}_{3.4 .5}(0.56 \mathrm{~g})$ was purified by silica gel FCC, eluting with $n$-hexane-EtOAc $(1: 1)$ to give $3(507.4 \mathrm{mg})$ as a colorless viscous liquid. Fraction $\mathrm{LH}_{4}(6.7 \mathrm{~g})$ was purified by silica gel FCC, eluting with acetone- $\mathrm{CH}_{2} \mathrm{Cl}_{2}(1: 4)$ to yield 3 subfractions, $\mathrm{LH}_{4.1}-\mathrm{LH}_{4.3}$. Subfraction $\mathrm{LH}_{4.3}(3.0 \mathrm{~g})$ was chromatographed on silica gel FCC, eluted with $n$-hexane-acetone ( $7: 3$ ) to give 3 subfractions, $\mathrm{LH}_{4.3 .1}-\mathrm{LH}_{4.3 .3}$. Solid in subfraction $\mathrm{LH}_{4.3 .3}(1.6 \mathrm{~g})$ was crystallized from $\mathrm{CH}_{2} \mathrm{Cl}_{2}-n$-hexane to give 12 (332 mg) as a yellow solid. Fraction $\mathrm{LH}_{5}(1.9 \mathrm{~g})$ was separated on silica gel FCC, eluted with $n$-hexane-acetone $(7: 3)$ to give 2 subfractions, $\mathrm{LH}_{5.1}-\mathrm{LH}_{5.2}$. Solid in subfraction $\mathrm{LH}_{5.2}(0.62 \mathrm{~g})$ was crystallized from $\mathrm{MeOH}-n$-hexane to give $10(30 \mathrm{mg})$ as colorless needles. The EtOAc extract was separated over silica gel FCC, using gradient elution with $n$-hexane-EtOAc ( $80: 20$ to $0: 100)$ and EtOAc-MeOH $(80: 20$ to $0: 100)$ to afford 6 fractions, $\mathrm{LE}_{1}-\mathrm{LE}_{6}$. Fraction $\mathrm{LE}_{2}(6.3 \mathrm{~g})$ was then purified by silica gel FCC, eluting with $n$-hexane-acetone $(4: 1)$ to give 2 subfractions, $\mathrm{LE}_{2.1}-\mathrm{LE}_{2.2}$. Further purification of subfraction $\mathrm{LE}_{2.2}$ $(0.74 \mathrm{~g})$ by silica gel FCC, eluting with $n$-hexane-EtOAc $(4: 1)$ gave 13 (552 mg) as a white solid. Fraction $\mathrm{LE}_{3}(3.6 \mathrm{~g})$ was purified by silica gel FCC, eluting with $n$-hexane-acetone $(3: 1)$ to give 2 subfractions, $\mathrm{LE}_{3.1}-\mathrm{LE}_{3.2}$. Subfraction $\mathrm{LE}_{3.2}(0.31 \mathrm{~g})$ was further separated by LiChroprep RP-18 column chromatography, eluting with $\mathrm{MeOH}-\mathrm{H}_{2} \mathrm{O}(6: 1)$ to give $16(17.6 \mathrm{mg})$ as a colorless viscous liquid. Fraction $\mathrm{LE}_{4}(12.0 \mathrm{~g})$ was separated by silica gel FCC, eluting with $n$-hexane-acetone $(7: 3)$ to give 4 subfractions, $\mathrm{LE}_{4.1}-\mathrm{LE}_{4.4}$. Subfraction $\mathrm{LE}_{4.1}(2.6 \mathrm{~g})$ was purified by LiChroprep RP-18 column chromatography, eluting with $\mathrm{MeOH}-\mathrm{H}_{2} \mathrm{O}(4: 1)$ to give 2 subfractions, $\mathrm{LE}_{4.1 .1}-\mathrm{LE}_{4.1 .2}$. Subfraction $\mathrm{LE}_{4.1 .1}(1.8 \mathrm{~g}$ ) was separated on silica gel FCC, eluting with $\mathrm{MeOH}-\mathrm{CH}_{2} \mathrm{Cl}_{2}(1: 19)$ to give 3 subfractions, $\mathrm{LE}_{4.1 .1 .1}$ $\mathrm{LE}_{\text {4.1.1.3. }}$ Solid in subfraction $\mathrm{LH}_{4.1 .1 .1}$ was crystallized from $\mathrm{MeOH}$ to give $12(21 \mathrm{mg})$ as a yellow solid. Subfraction $\mathrm{LE}_{\text {4.1.1.2 }}$
(1.4 g) was purified by silica gel FCC, eluting with $n$-hexaneEtOAc $(1: 1)$ to give an additional amount of 2 (214 mg). Subfraction $\mathrm{LE}_{4.1 .2}(0.67 \mathrm{~g})$ was purified by silica gel FCC, eluting with $n$-hexane-acetone $(7: 3)$ to give $5(25.8 \mathrm{mg})$ as a colorless viscous liquid. Subfraction $\mathrm{LE}_{4.2}(3.0 \mathrm{~g})$ was purified by silica gel FCC, eluting with $n$-hexane-acetone $(7: 3)$ to give 2 subfractions, $\mathrm{LE}_{4.2 .1}-\mathrm{LE}_{4.2 .2}$. Solid in subfraction $\mathrm{LH}_{4.2 .1}$ was crystallized from $\mathrm{MeOH}$ to give an additional amount of 12 (105.3 $\mathrm{mg})$. Solid in subfraction $\mathrm{LH}_{4.2 .2}(0.54 \mathrm{~g})$ was crystallized from $\mathrm{MeOH}$ to give 14 (30 mg) as a white solid. Subfraction $\mathrm{LE}_{4.3}(2.9$ g) was purified by silica gel FCC, eluting with $n$-hexane-EtOAc $(1: 1)$, to give an additional amount of $3(174 \mathrm{mg})$. Solid in subfraction $\mathrm{LE}_{4.4}(0.12 \mathrm{~g})$ was crystallized from $\mathrm{DMF}-\mathrm{CH}_{2} \mathrm{Cl}_{2}$ to give 15 (26 mg) as a yellow solid. Fraction $\mathrm{LE}_{5}(8.4 \mathrm{~g})$ was purified by silica gel FCC, eluting with $\mathrm{MeOH}-\mathrm{CH}_{2} \mathrm{Cl}_{2}(3: 97)$ to give 2 subfractions, $\mathrm{LE}_{5.1}-\mathrm{LE}_{5.2}$. Solid in subfraction $\mathrm{LE}_{5.1}(1.6 \mathrm{~g})$ was crystallized from $\mathrm{MeOH}-\mathrm{CH}_{2} \mathrm{Cl}_{2}$ to give $10(1.08 \mathrm{~g})$ as colorless needles and the filtrate was further purified by silica gel FCC, eluting with EtOAc- $\mathrm{CH}_{2} \mathrm{Cl}_{2}(15: 85)$ to yield $9(22 \mathrm{mg})$ as a white solid and $\mathbf{1 1}(75 \mathrm{mg})$ as colorless needles. Solids in subfractions $\mathrm{LE}_{5.2}(6.2 \mathrm{~g})$ and $\mathrm{LE}_{6}(10.9 \mathrm{~g})$ were crystallized from $\mathrm{DMF}-\mathrm{CH}_{2} \mathrm{Cl}_{2}$ to give 15 (931 $\mathrm{mg})$ as a yellow solid.

2.3.1 Velutinone A (1). Colorless viscous liquid; $R_{\mathrm{f}}=0.39$ ( $n$-hexane-EtOAc, $7: 3) ;[\alpha]_{\mathrm{D}}^{23}-68.0\left(c 0.20, \mathrm{CHCl}_{3}\right)$; $\mathrm{ECD}(80 \mu \mathrm{M}$, $\mathrm{MeOH}) \lambda_{\max }(\Delta \varepsilon) 215(-19.02) \mathrm{nm}$; IR (ATR) $\nu_{\max } 2966,2916$, 2854, 1781, 1685, 1440, 1418, 1383, 1161, and $997 \mathrm{~cm}^{-1}$; for ${ }^{1} \mathrm{H}$ and ${ }^{13} \mathrm{C}$ NMR spectroscopic data, see Table 1 ; HRESITOFMS $\mathrm{m} / \mathrm{z}$ 311.1611 [M $+\mathrm{Na}]^{+}$(calcd for $\mathrm{C}_{18} \mathrm{H}_{24} \mathrm{O}_{3}+\mathrm{Na}, 311.1618$ ).

2.3.2 Velutinone B (2). Colorless viscous liquid; $R_{\mathrm{f}}=0.34$ ( $n$-hexane-EtOAc, $1: 1) ;[\alpha]_{\mathrm{D}}^{24}-61.2$ (c $\left.0.20, \mathrm{CHCl}_{3}\right)$; ECD (130 $\mu \mathrm{M}, \mathrm{MeOH}) \lambda_{\max }(\Delta \varepsilon) 215(-28.90) \mathrm{nm}$; IR (ATR) $\nu_{\max } 3468,2920$, $2857,1777,1683,1447,1385,1165,1065$, 995, and $990 \mathrm{~cm}^{-1}$; for ${ }^{1} \mathrm{H}$ and ${ }^{13} \mathrm{C}$ NMR spectroscopic data, see Table 1 ; HRESITOFMS $m / z$ 305.1740 $[\mathrm{M}+\mathrm{H}]^{+}$(calcd for $\mathrm{C}_{18} \mathrm{H}_{24} \mathrm{O}_{4}+\mathrm{H}^{+}, 305.1747$ ).

2.3.3 Velutinone C (3). Colorless viscous liquid; $R_{\mathrm{f}}=0.29$ ( $n$-hexane-EtOAc, $1: 1)$; $[\alpha]_{\mathrm{D}}^{23}-55.4\left(c 0.20, \mathrm{CHCl}_{3}\right)$; $\operatorname{ECD}(50 \mu \mathrm{M}$, $\mathrm{MeOH}) \lambda_{\max }(\Delta \varepsilon) 213(-7.63) \mathrm{nm}$; IR (ATR) $\nu_{\max } 3447,2972$, 2929, 1778, 1682, 1481, 1385, 1155, and $975 \mathrm{~cm}^{-1}$; for ${ }^{1} \mathrm{H}$ and ${ }^{13} \mathrm{C}$ NMR spectroscopic data, see Table 1 ; HRESITOFMS $\mathrm{m} / \mathrm{z}$ $327.1543[\mathrm{M}+\mathrm{Na}]^{+}$(calcd for $\mathrm{C}_{18} \mathrm{H}_{24} \mathrm{O}_{4}+\mathrm{Na}, 327.1572$ ).

2.3.4 Velutinone $\mathrm{D}(4)$. Colorless viscous liquid; $R_{\mathrm{f}}=0.39$ ( $n$-hexane-EtOAc, $1: 1)$; $[\alpha]_{\mathrm{D}}^{24}-58.4\left(c 0.20, \mathrm{CHCl}_{3}\right)$; $\operatorname{ECD}(56 \mu \mathrm{M}$, $\mathrm{MeOH}) \lambda_{\max }(\Delta \varepsilon) 213(-9.74) \mathrm{nm}$; IR (ATR) $\nu_{\max } 3393,2978$, 2931, 1778, 1682, 1417, 1384, 1165, and $996 \mathrm{~cm}^{-1}$; for ${ }^{1} \mathrm{H}$ and ${ }^{13} \mathrm{C}$ NMR spectroscopic data, see Table 1; HRESITOFMS $\mathrm{m} / \mathrm{z}$ 343.1472 $[\mathrm{M}+\mathrm{Na}]^{+}$(calcd for $\mathrm{C}_{18} \mathrm{H}_{24} \mathrm{O}_{5}+\mathrm{Na}, 343.1521$ ).

2.3.5 Velutinone $\mathrm{E}$ (5). Colorless viscous liquid; $R_{\mathrm{f}}=0.37$ ( $n$-hexane-EtOAc, $1: 1)$; $[\alpha]_{\mathrm{D}}^{23}-32.5\left(c 0.20, \mathrm{CHCl}_{3}\right)$; $\mathrm{ECD}(62 \mu \mathrm{M}$, $\mathrm{MeOH}) \lambda_{\max }(\Delta \varepsilon) 202(-13.84) \mathrm{nm}$; IR (ATR) $\nu_{\max } 3442,3030$, 2967, 2921, 2856, 1771, 1668, 1446, 1419, 1377, 1328, 1167, 1069, 1030, and $990 \mathrm{~cm}^{-1}$; for ${ }^{1} \mathrm{H}$ and ${ }^{13} \mathrm{C}$ NMR spectroscopic data, see Table 2; HRESITOFMS $m / z$ 291.1935 [M + H] $]^{+}$(calcd for $\left.\mathrm{C}_{18} \mathrm{H}_{26} \mathrm{O}_{3}+\mathrm{H}^{+}, 291.1955\right)$.

2.3.6 Velutinone $\mathbf{F}$ (6). Colorless viscous liquid; $R_{\mathrm{f}}=0.50$ ( $n$-hexane-EtOAc, $7: 3) ;[\alpha]_{\mathrm{D}}^{23}-29.5\left(c 0.20, \mathrm{CHCl}_{3}\right)$; $\operatorname{ECD}(72 \mu \mathrm{M}$, $\mathrm{MeOH}) \lambda_{\max }(\Delta \varepsilon) 207(+4.83) \mathrm{nm}$; IR (ATR) $\nu_{\max } 2967,2917,2855$, $1789,1719,1423,1377,1347,1161,1035,857$, and $802 \mathrm{~cm}^{-1}$; for 
Table $1{ }^{1} \mathrm{H}$ and ${ }^{13} \mathrm{C}$ NMR spectroscopic data of $1-4$ in $\mathrm{CDCl}_{3}$

\begin{tabular}{|c|c|c|c|c|c|c|c|c|}
\hline \multirow[b]{2}{*}{ No. } & \multicolumn{2}{|l|}{1} & \multicolumn{2}{|l|}{2} & \multicolumn{2}{|l|}{3} & \multicolumn{2}{|l|}{4} \\
\hline & $\delta_{\mathrm{C}}$ & $\delta_{\mathrm{H}}(J$ in $\mathrm{Hz})$ & $\delta_{\mathrm{C}}$ & $\delta_{\mathrm{H}}(J$ in $\mathrm{Hz})$ & $\delta_{\mathrm{C}}$ & $\delta_{\mathrm{H}}(J$ in $\mathrm{Hz})$ & $\delta_{\mathrm{C}}$ & $\delta_{\mathrm{H}}(J$ in $\mathrm{Hz})$ \\
\hline 1 & 174.2 & & 174.3 & & 174.2 & & 174.4 & \\
\hline 2 & 38.9 & $\begin{array}{l}\text { 2.48, d (17.4), } \\
\mathrm{H} \alpha / 2.39, \mathrm{~d}(17.4), \mathrm{H} \beta\end{array}$ & 38.9 & $\begin{array}{l}2.43, \mathrm{~d}(17.8) \\
\mathrm{H} \alpha / 2.39, \mathrm{~d}(17.8), \mathrm{H} \beta\end{array}$ & 39.0 & $2.40, \mathrm{~s}$ & 39.0 & $2.42, \mathrm{~s}$ \\
\hline $2 \mathrm{a}$ & 44.7 & & 44.6 & & 44.5 & & 44.5 & \\
\hline 3 & 42.6 & $2.52, \mathrm{~s}$ & 42.7 & $\begin{array}{l}2.55, \mathrm{~d}(16.7) \\
\mathrm{H} \alpha / 2.50, \mathrm{~d}(16.7), \mathrm{H} \beta\end{array}$ & 42.9 & $\begin{array}{l}2.53, \mathrm{~d}(16.7) \\
\mathrm{H} \alpha / 2.48, \mathrm{~d}(16.7), \mathrm{H} \beta\end{array}$ & 43.0 & $\begin{array}{l}2.56, \mathrm{~d}(16.7) \\
\mathrm{H} \alpha / 2.50, \mathrm{~d}(16.7), \mathrm{H} \beta\end{array}$ \\
\hline 4 & 196.2 & & 196.2 & & 196.1 & & 196.3 & \\
\hline 5 & 131.2 & $6.17, \mathrm{dd}(10.3,1.2)$ & 131.2 & $6.13, \mathrm{dd}(10.3,1.2)$ & 131.2 & $6.12, \mathrm{dd}(10.3,1.2)$ & 131.2 & $6.18, \mathrm{dd}(10.3,1.2)$ \\
\hline 6 & 141.4 & 6.75 , dd $(10.3,3.3)$ & $141.4^{a}$ & 6.74, dd $(10.3,3.3)$ & 141.3 & 6.74, dd $(10.3,3.3)$ & 141.4 & 6.76, dd $(10.3,3.3)$ \\
\hline $6 \mathrm{a}$ & 77.8 & 4.87, dd $(3.3,1.2)$ & 77.9 & $4.87, \mathrm{~d}(3.3)$ & 77.9 & $4.86, \mathrm{dd},(3.3,1.2)$ & 78.1 & $4.88, \mathrm{dd},(3.3,1.2)$ \\
\hline $1^{\prime}$ & 36.0 & $\begin{array}{l}2.31, \mathrm{dd}(14.4,7.7), \\
\mathrm{Ha} / 2.21, \\
\mathrm{dd}(14.4,7.7), \mathrm{Hb}\end{array}$ & 36.2 & $\begin{array}{l}2.31, \text { dd }(14.5,7.7), \\
\mathrm{Ha} / 2.22 \text {, dd }(14.5,7.7), \\
\mathrm{Hb}\end{array}$ & 36.6 & $\begin{array}{l}2.30, \text { dd }(14.4,7.7), \\
\mathrm{Ha} / 2.23 \text {, dd }(14.4,7.7) \text {, } \\
\mathrm{Hb}\end{array}$ & 36.8 & $\begin{array}{l}2.32 \text {, dd }(14.4,7.7), \\
\mathrm{Ha} / 2.26 \text {, dd }(14.4,7.7), \\
\mathrm{Hb}\end{array}$ \\
\hline $2^{\prime}$ & 116.9 & $5.10, \operatorname{td}(7.7,1.4)$ & 116.9 & $5.15, \operatorname{td}(7.7,1.4)$ & 117.5 & $5.11, \operatorname{td}(7.7,1.4)$ & 117.8 & $5.15, \operatorname{td}(7.7,1.4)$ \\
\hline $3^{\prime}$ & 141.5 & & $141.4^{a}$ & & 140.3 & & 140.0 & \\
\hline $4^{\prime}$ & 39.9 & $2.10-2.02, \mathrm{~m}^{a}$ & 35.9 & $2.14-1.98, \mathrm{~m}$ & 42.4 & $2.68, \mathrm{~d}(6.5)$ & 42.5 & $2.73, \mathrm{~d}(5.5)$ \\
\hline $5^{\prime}$ & 26.2 & $2.10-2.02, \mathrm{~m}^{a}$ & 33.0 & $1.65-1.58, \mathrm{~m}$ & 123.8 & $5.51, \mathrm{dt}(15.6,6.6)$ & 128.0 & $5.62, \mathrm{dt}(15.8,6.0)$ \\
\hline $6^{\prime}$ & 123.7 & $5.01, \mathrm{t}(5.4)$ & 75.3 & $4.00, \mathrm{t}(6.3)$ & 140.2 & $5.60, \mathrm{~d},(15.6)$ & 135.9 & $5.56, \mathrm{~d},(15.8)$ \\
\hline $7^{\prime}$ & 131.9 & & 147.3 & & 70.4 & & 81.8 & \\
\hline $8^{\prime}$ & 25.7 & $1.65, \mathrm{~s}$ & 111.1 & $\begin{array}{l}\text { 4.91, brs, } \\
\mathrm{Ha} / 4.82, \mathrm{t}(1.4), \mathrm{Hb}\end{array}$ & 29.7 & $1.28, \mathrm{~s}$ & 24.3 & $1.31, \mathrm{~s}$ \\
\hline $9^{\prime}$ & 17.7 & $1.58, \mathrm{~s}$ & 17.5 & $1.70, \mathrm{~s}$ & 29.7 & $1.28, \mathrm{~s}$ & 24.2 & $1.31, \mathrm{~s}$ \\
\hline $10^{\prime}$ & 16.3 & $1.60, \mathrm{~s}$ & 16.4 & $1.61, \mathrm{~s}$ & 16.5 & $1.58, \mathrm{~s}$ & 16.6 & $1.60, \mathrm{~s}$ \\
\hline
\end{tabular}

Table $2{ }^{1} \mathrm{H}$ and ${ }^{13} \mathrm{C}$ NMR spectroscopic data of $5-8$ in $\mathrm{CDCl}_{3}$

\begin{tabular}{|c|c|c|c|c|c|c|c|c|}
\hline \multirow[b]{2}{*}{ No. } & \multicolumn{2}{|l|}{5} & \multicolumn{2}{|l|}{6} & \multicolumn{2}{|l|}{7} & \multicolumn{2}{|l|}{8} \\
\hline & $\delta_{\mathrm{C}}$ & $\delta_{\mathrm{H}}(J$ in $\mathrm{Hz})$ & $\delta_{\mathrm{C}}$ & $\delta_{\mathrm{H}}(J$ in $\mathrm{Hz})$ & $\delta_{\mathrm{C}}$ & $\delta_{\mathrm{H}}(J$ in $\mathrm{Hz})$ & $\delta_{\mathrm{C}}$ & $\delta_{\mathrm{H}}(J$ in $\mathrm{Hz})$ \\
\hline 1 & 176.2 & & 173.8 & & 173.9 & & 173.7 & \\
\hline 2 & $37.1^{a}$ & $\begin{array}{l}2.38, \mathrm{~d}(17.4) \\
\mathrm{H} \alpha / 2.19 \\
\mathrm{~d}(17.4), \mathrm{H} \beta\end{array}$ & 38.3 & $\begin{array}{l}2.32, \mathrm{~d}(17.4) \\
\mathrm{H} \alpha / 2.28 \\
\mathrm{~d}(17.4), \mathrm{H} \beta\end{array}$ & 38.4 & $2.30-2.24, \mathrm{~m}^{a}$ & 38.4 & $2.30-2.24, \mathrm{~m}^{a}$ \\
\hline $2 \mathrm{a}$ & 42.3 & & 47.5 & & 47.4 & & 47.4 & \\
\hline 3 & $37.2^{a}$ & $\begin{array}{l}2.25-2.17, \mathrm{~m}^{a}, \\
\mathrm{H} \alpha / 1.58-1.51 \\
\mathrm{~m}, \mathrm{H} \beta\end{array}$ & $39.9^{a}$ & $\begin{array}{l}\text { 2.86, d (13.9), } \\
\mathrm{H} \alpha / 2.14, \\
\mathrm{~d}(13.9), \mathrm{H} \beta\end{array}$ & 39.9 & $\begin{array}{l}2.82, \mathrm{~d}(13.9) \\
\mathrm{H} \alpha / 2.11 \\
\mathrm{~d}(13.9), \mathrm{H} \beta\end{array}$ & 39.9 & $\begin{array}{l}\text { 2.84, d (13.9), } \\
\mathrm{H} \alpha / 2.12, \\
\mathrm{~d}(13.9), \mathrm{H} \beta\end{array}$ \\
\hline 4 & 63.7 & $4.31, \mathrm{~m}$ & 204.6 & & 204.5 & & 204.5 & \\
\hline 5 & 135.2 & 5.97, brd $(10.2)$ & 58.6 & $3.65, \mathrm{~d}(3.6)$ & 58.5 & $3.62, \mathrm{~d}(3.6)$ & 58.5 & $3.62, \mathrm{~d}(3.7)$ \\
\hline 6 & 125.2 & $6.75-5.65, \mathrm{~m}$ & 54.9 & $3.37, \mathrm{~d}(3.6)$ & 54.8 & $3.34, \mathrm{~d}(3.6)$ & 54.8 & $3.34, \mathrm{~d}(3.7)$ \\
\hline $6 \mathrm{a}$ & 80.1 & $4.62, \mathrm{~m}$ & 76.6 & $4.71, \mathrm{~d}(3.6)$ & 76.6 & $4.68, \mathrm{~s}$ & 76.5 & $4.69, \mathrm{~s}$ \\
\hline $1^{\prime}$ & 36.5 & $2.25-2.09, \mathrm{~m}$ & 36.9 & $\begin{array}{l}2.29, \text { dd }(14.4,7.7) \\
\mathrm{Ha} / 2.20, \\
\text { dd }(14.4,7.7), \mathrm{Hb}\end{array}$ & 36.8 & $2.25-2.09, \mathrm{~m}^{a}$ & $36.9^{a}$ & $2.32-2.09, \mathrm{~m}^{a}$ \\
\hline $2^{\prime}$ & 117.5 & $5.12, \operatorname{td}(7.7,1.4)$ & 116.2 & $5.08, \operatorname{td}(7.7,1.4)$ & 116.3 & $5.10, \operatorname{td}(7.8,1.4)$ & 116.7 & $5.14, \operatorname{td}(7.7,1.4)$ \\
\hline $3^{\prime}$ & 140.2 & & 141.8 & & 141.6 & & 141.1 & \\
\hline $4^{\prime}$ & 39.8 & $2.08-1.98, \mathrm{~m}^{a}$ & $39.9^{a}$ & $2.10-2.02, \mathrm{~m}^{a}$ & 35.8 & $2.01-1.96, \mathrm{~m}^{a}$ & $36.7^{a}$ & $2.32-2.09, \mathrm{~m}^{a}$ \\
\hline $5^{\prime}$ & 26.3 & $2.08-1.98, \mathrm{~m}^{a}$ & 26.2 & $2.10-2.02, \mathrm{~m}^{a}$ & 32.8 & & & \\
\hline $6^{\prime}$ & 123.8 & $5.01, \mathrm{~m}$ & 123.6 & $5.04, \mathrm{t}(6.6)$ & 75.1 & $1.64-1.55, \mathrm{~m}$ & 27.2 & $1.69-1.53, \mathrm{~m}$ \\
\hline $7^{\prime}$ & 131.6 & & 132.0 & & 147.3 & $3.98, \mathrm{t}(6.0)$ & 63.7 & $3.65, \mathrm{t}(6.7)$ \\
\hline $8^{\prime}$ & 25.6 & $1.63, \mathrm{~s}$ & 25.7 & $1.68, \mathrm{~s}$ & 111.0 & $\begin{array}{l}4.79, \text { brs, } \\
\mathrm{Ha} / 4.89, \mathrm{t}(0.8), \mathrm{Hb}\end{array}$ & 58.2 & \\
\hline $9^{\prime}$ & 17.6 & $1.56, \mathrm{~s}$ & 17.7 & $1.59, \mathrm{~s}$ & 17.5 & $1.68, \mathrm{~s}$ & 24.7 & $1.27, \mathrm{~s}$ \\
\hline $10^{\prime}$ & 16.2 & $1.58, \mathrm{~s}$ & 16.4 & $1.61, \mathrm{~s}$ & 16.4 & $1.60, \mathrm{~s}$ & 18.7 & $1.23, \mathrm{~s}$ \\
\hline
\end{tabular}


${ }^{1} \mathrm{H}$ and ${ }^{13} \mathrm{C}$ NMR spectroscopic data, see Table 2; HRESITOFMS $m / z 327.1562[\mathrm{M}+\mathrm{Na}]^{+}$(calcd for $\mathrm{C}_{18} \mathrm{H}_{24} \mathrm{O}_{4}+\mathrm{Na}, 327.1567$ ).

2.3.7 Velutinone $\mathbf{G}$ (7). Colorless viscous liquid; $R_{\mathrm{f}}=0.42$ (hexane-EtOAc, $1: 3$ ); $[\alpha]_{\mathrm{D}}^{23}-25.6\left(c 0.20, \mathrm{CHCl}_{3}\right)$; ECD $(94 \mu \mathrm{M}$, $\mathrm{MeOH}) \lambda_{\max }(\Delta \varepsilon) 205(+11.89) \mathrm{nm}$; IR (ATR) $\nu_{\max } 3481,2939,2858$, 1783, 1718, 1651, 1422, 1347, 1164, 1033, and $902 \mathrm{~cm}^{-1}$ for ${ }^{1} \mathrm{H}$ and ${ }^{13} \mathrm{C}$ NMR spectroscopic data, see Table 2; HRESITOFMS $\mathrm{m} / \mathrm{z}$ $321.1690[\mathrm{M}+\mathrm{H}]^{+}$(calcd for $\mathrm{C}_{18} \mathrm{H}_{24} \mathrm{O}_{5}+\mathrm{H}^{+}, 321.1697$ ).

2.3.8 Velutinone $\mathbf{H}$ (8). Colorless viscous liquid; $R_{\mathrm{f}}=0.29$ (n-hexane-EtOAc, $7: 3)$; $[\alpha]_{\mathrm{D}}^{24}-23.5\left(c 0.20, \mathrm{CHCl}_{3}\right)$; $\operatorname{ECD}(75 \mu \mathrm{M}$, $\mathrm{MeOH}) \lambda_{\max }(\Delta \varepsilon) 215(+11.84) \mathrm{nm}$; IR (ATR) $\nu_{\max } 2962,2924$, $2855,1785,1719,1423,1378,1163$, and $1034 \mathrm{~cm}^{-1}$; for ${ }^{1} \mathrm{H}$ and ${ }^{13} \mathrm{C}$ NMR spectroscopic data, see Table 2; HRESITOFMS $\mathrm{m} / \mathrm{z}$ 321.1690 $[\mathrm{M}+\mathrm{H}]^{+}$(calcd for $\mathrm{C}_{18} \mathrm{H}_{24} \mathrm{O}_{5}+\mathrm{H}^{+}, 321.1697$ ).

2.3.9 Velutindimer A (9). White solid; $\mathrm{mp} 201-203{ }^{\circ} \mathrm{C} ; R_{\mathrm{f}}=$ 0.13 (n-hexane-EtOAc, $1: 1) ;[\alpha]_{\mathrm{D}}^{28}+0.08$ (c 0.63, $\mathrm{MeOH}-\mathrm{CHCl}_{3}$, $3: 1)$; ECD $(26 \mu \mathrm{M}, \mathrm{MeOH}) \lambda_{\max }(\Delta \varepsilon) 285(0.00) \mathrm{nm}$; UV (MeOH) $\lambda_{\max }(\log \varepsilon) 226$ (4.53), 285 (4.20); IR (ATR) $\nu_{\max } 3088,2944,2837$, $1715,1643,1611,1564,1513,1455,1409,1249,1180,1034$, and $818 \mathrm{~cm}^{-1}$; for ${ }^{1} \mathrm{H}$ and ${ }^{13} \mathrm{C}$ NMR spectroscopic data, see Table 3; HRESITOFMS $m / z 539.1651[\mathrm{M}+\mathrm{Na}]^{+}$(calcd for $\mathrm{C}_{30} \mathrm{H}_{28} \mathrm{O}_{8}+\mathrm{Na}$, 539.1682).

2.3.10 Velutinin dimer B (10). Colorless needles; $\mathrm{mp} 205-$ $207{ }^{\circ} \mathrm{C} ; R_{\mathrm{f}}=0.21$ ( $n$-hexane-EtOAc, $\left.1: 1\right) ;[\alpha]_{\mathrm{D}}^{28}+0.08(c 0.63$,
$\left.\mathrm{MeOH}-\mathrm{CHCl}_{3}, 5: 3\right) ; \mathrm{ECD}(33 \mu \mathrm{M}, \mathrm{MeOH}) \lambda_{\max }(\Delta \varepsilon) 265$ (0.00) nm; UV (MeOH) $\lambda_{\max }(\log \varepsilon) 265$ (4.45); IR (ATR) $\nu_{\max } 2940$, 2838, 1699, 1647, 1608, 1566, 1512, 1455, 1410, 1391, 1246, 1176, 1031 and $814 \mathrm{~cm}^{-1}$; for ${ }^{1} \mathrm{H}$ and ${ }^{13} \mathrm{C}$ NMR spectroscopic data, see Table 3; HRESITOFMS $m / z$ 539.1666 [M $+\mathrm{Na}]^{+}$(calcd for $\left.\mathrm{C}_{30} \mathrm{H}_{28} \mathrm{O}_{8}+\mathrm{Na}, 539.1682\right)$.

2.3.11 Velutinin dimer $\mathbf{C}$ (11). Colorless needles; $\mathrm{mp}$ 207$209{ }^{\circ} \mathrm{C} ; R_{\mathrm{f}}=0.18$ (n-hexane-EtOAc, $\left.1: 1\right) ;[\alpha]_{\mathrm{D}}^{27}+0.03(c 0.23$, $\left.\mathrm{MeOH}-\mathrm{CHCl}_{3}, 9: 1\right)$; ECD $(22 \mu \mathrm{M}, \mathrm{MeOH}) \lambda_{\max }(\Delta \varepsilon) 268(0.00)$ UV (MeOH) $\lambda_{\max }(\log \varepsilon) 268$ (4.59); IR (ATR) $\nu_{\max } 2924,2837$, 1708, 1649, 1625, 1608, 1567, 1513, 1455, 1406, 1248, 1176, 1031, and $821 \mathrm{~cm}^{-1}$; for ${ }^{1} \mathrm{H}$ and ${ }^{13} \mathrm{C}$ NMR spectroscopic data, see Table 3; HRESITOFMS $m / z 539.1664[\mathrm{M}+\mathrm{Na}]^{+}$(calcd for $\left.\mathrm{C}_{30} \mathrm{H}_{28} \mathrm{O}_{8}+\mathrm{Na}, 539.1682\right)$.

\subsection{Preparation of the $(R)$-and $(S)$ - $\alpha$-methoxy- $\alpha$ - (trifluoromethyl) phenyl acetate of 2}

The determination of configuration for the stereogenic carbinol carbons was carried out following the method reported by Ohtani et al. ${ }^{22}$ A solution of $(S)$-MPTA-Cl $(10 \mu \mathrm{L}, 53.4 \mu \mathrm{mol})$ was added to a solution mixture of $2(8 \mathrm{mg}, 27.5 \mu \mathrm{mol})$ and DMAP (5 $\mathrm{mg})$ in dry $\mathrm{CH}_{2} \mathrm{Cl}_{2}(1 \mathrm{~mL})$ and stirred under $\mathrm{N}_{2}$ at room temperature for $6 \mathrm{~h}$. Then the solvent was removed in vacuo. The

Table $3{ }^{1} \mathrm{H}$ and ${ }^{13} \mathrm{C}$ NMR spectroscopic data of 9-11 in $\mathrm{CDCl}_{3}$

\begin{tabular}{|c|c|c|c|c|c|c|}
\hline \multirow[b]{2}{*}{ No. position } & \multicolumn{2}{|l|}{9} & \multicolumn{2}{|l|}{10} & \multicolumn{2}{|l|}{11} \\
\hline & $\delta_{\mathrm{C}}$ & $\delta_{\mathrm{H}}(J$ in $\mathrm{Hz})$ & $\delta_{\mathrm{C}}$ & $\delta_{\mathrm{H}}(J$ in $\mathrm{Hz})$ & $\delta_{\mathrm{C}}$ & $\delta_{\mathrm{H}}(J$ in $\mathrm{Hz})$ \\
\hline 2 & 164.0 & & 163.9 & & 164.1 & \\
\hline 3 & 87.7 & $5.21, \mathrm{~d}(2.2)$ & 88.6 & $5.33, \mathrm{~d}(2.2)$ & 88.9 & $5.45, \mathrm{~d}(2.2)$ \\
\hline 4 & 170.1 & & 170.5 & & 170.6 & \\
\hline 5 & 101.3 & $5.71, \mathrm{~d}(2.2)$ & 102.5 & $5.89, \mathrm{~d}(2.2)$ & 102.0 & $5.99, \mathrm{~d}(2.2)$ \\
\hline 6 & 162.9 & & 158.9 & & 158.7 & \\
\hline 7 & 43.0 & 4.35, dd $(10.0,7.6)$ & 55.0 & 4.09, d (10.8) & 54.5 & $3.64, \mathrm{~d}(10.3)$ \\
\hline 8 & 45.5 & 4.16, dd $(10.0,7.6)$ & 38.7 & 4.26, dd $(10.8,9.9)$ & 45.6 & 4.00, dd $(10.3,9.7)$ \\
\hline 9 & 129.4 & & 127.7 & & 131.4 & \\
\hline 10 & 128.5 & $7.19, \mathrm{~d}(8.7)$ & 128.6 & $7.16, \mathrm{~d}(8.7)$ & 127.4 & $7.22, \mathrm{~d}(8.6)$ \\
\hline 11 & 113.9 & $6.82, \mathrm{~d}(8.7)$ & 113.8 & $6.85, \mathrm{~d}(8.7)$ & 114.2 & $6.89, \mathrm{~d}(8.6)$ \\
\hline 12 & 158.6 & & 159.2 & & 159.0 & \\
\hline 13 & 113.9 & $6.82, \mathrm{~d}(8.7)$ & 113.8 & $6.85, \mathrm{~d}(8.7)$ & 114.2 & $6.89, \mathrm{~d}(8.6)$ \\
\hline 14 & 128.5 & $7.19, \mathrm{~d}(8.7)$ & 128.6 & $7.16, \mathrm{~d}(8.7)$ & 127.4 & $7.22, \mathrm{~d}(8.6)$ \\
\hline $2^{\prime}$ & 164.0 & & 164.7 & & 165.4 & \\
\hline $3^{\prime}$ & 87.7 & $5.21, \mathrm{~d}(2.2)$ & 91.7 & $5.29, \mathrm{~s}$ & 89.2 & 5.19, s \\
\hline $4^{\prime}$ & 170.1 & & 170.1 & & 171.4 & \\
\hline $5^{\prime}$ & 101.3 & $5.71, \mathrm{~d}(2.2)$ & 45.8 & $3.55, \mathrm{~d}(9.9)$ & 44.2 & $3.20, \mathrm{~d}(9.7)$ \\
\hline $6^{\prime}$ & 162.9 & & 79.4 & & 82.6 & \\
\hline $7^{\prime}$ & 43.0 & 4.35, dd $(10.0,7.6)$ & 122.2 & $6.42, \mathrm{~d}(15.8)$ & 125.2 & $6.18, \mathrm{~d}(15.9)$ \\
\hline $8^{\prime}$ & 45.5 & 4.16, dd $(10.0,7.6)$ & 130.8 & $6.86, \mathrm{~d}(15.8)$ & 131.3 & $6.63, \mathrm{~d}(15.9)$ \\
\hline $9^{\prime}$ & 129.4 & & 127.7 & & 127.9 & \\
\hline $10^{\prime}$ & 128.5 & $7.19, \mathrm{~d}(8.7)$ & 128.1 & $7.34, \mathrm{~d}(8.7)$ & 128.8 & $7.33, \mathrm{~d}(8.7)$ \\
\hline $11^{\prime}$ & 113.9 & $6.82, \mathrm{~d}(8.7)$ & 114.1 & $6.85, \mathrm{~d}(8.7)$ & 114.0 & $6.84, \mathrm{~d}(8.7)$ \\
\hline $12^{\prime}$ & 158.6 & & 159.7 & & 159.9 & \\
\hline $13^{\prime}$ & 113.9 & $6.82, \mathrm{~d}(8.7)$ & 114.1 & $6.85, \mathrm{~d}(8.7)$ & 114.0 & $6.84, \mathrm{~d}(8.7)$ \\
\hline $14^{\prime}$ & 128.5 & $7.19, \mathrm{~d}(8.7)$ & 128.1 & $7.34, \mathrm{~d}(8.7)$ & 128.8 & $7.33, \mathrm{~d}(8.7)$ \\
\hline 4-OMe & 55.7 & $3.75, \mathrm{~s}$ & 55.8 & $3.69, \mathrm{~s}$ & 55.8 & $3.76, \mathrm{~s}$ \\
\hline 12-OMe & 55.2 & $3.67, \mathrm{~s}$ & 55.3 & $3.79, \mathrm{~s}$ & 55.27 & $3.79, \mathrm{~s}$ \\
\hline $4^{\prime}$-OMe & 55.7 & $3.75, \mathrm{~s}$ & 55.5 & $3.32, \mathrm{~s}$ & 56.1 & $3.77, \mathrm{~s}$ \\
\hline $12^{\prime}$-OMe & 55.2 & $3.67, \mathrm{~s}$ & 55.3 & $3.78, \mathrm{~s}$ & 55.30 & $3.80, \mathrm{~s}$ \\
\hline
\end{tabular}


residue was separated on preparative TLC $\left(\mathrm{CH}_{2} \mathrm{Cl}_{2}\right)$ to give the $(R)$-ester (2a, $7 \mathrm{mg}, 50 \%)$. The $(S)$-ester of 2 was prepared using the procedure described above [alcohol $2(10 \mathrm{mg}, 34.4 \mu \mathrm{mol})$, $\mathrm{CH}_{2} \mathrm{Cl}_{2}(1 \mathrm{~mL})$, dimethylaminopyridine (5 mg), and $(R)$-MPTA-Cl $(10 \mu \mathrm{L}, 53.4 \mu \mathrm{mol})]$ to yield $(S)$-ester $(2 \mathrm{~b}, 12 \mathrm{mg}, 71 \%)$.

2.4.1 Compound 2a. Colorless viscous liquid; ${ }^{1} \mathrm{H}$ NMR $\left(\mathrm{CDCl}_{3}, 400 \mathrm{MHz}\right) \delta 6.67(1 \mathrm{H}, \mathrm{dd}, J=10.3,3.3 \mathrm{~Hz}, \mathrm{H}-6), 6.19(1 \mathrm{H}$, $\mathrm{dd}, J=10.3,1.2 \mathrm{~Hz}, \mathrm{H}-5), 5.37\left(1 \mathrm{H}, \mathrm{dt}, J=12.0,6.4,6.4 \mathrm{~Hz}, \mathrm{H}-6^{\prime}\right)$, $5.07\left(1 \mathrm{H}, \mathrm{dt}, J=7.6,1.1 \mathrm{~Hz}, \mathrm{H}-2^{\prime}\right), 5.02$ (brs, H-8' a), 4.98 (1H, brs, $\left.\mathrm{H}-8^{\prime} \mathrm{b}\right), 4.87(1 \mathrm{H}, \mathrm{dd}, J=3.3,1.2, \mathrm{H}-6 \mathrm{a}), 2.56(1 \mathrm{H}, \mathrm{d}, J=16.7 \mathrm{~Hz}$, $\mathrm{H}-3 \alpha), 2.51(1 \mathrm{H}, \mathrm{d}, J=16.6 \mathrm{~Hz}, \mathrm{H}-3 \beta), 2.46(1 \mathrm{H}, \mathrm{d}, J=17.4 \mathrm{~Hz}, \mathrm{H}-$ $2 \alpha), 2.39(1 \mathrm{H}, \mathrm{d}, J=17.4 \mathrm{~Hz}, \mathrm{H}-2 \beta), 2.32(1 \mathrm{H}, \mathrm{dd}, J=14.0,7.7 \mathrm{~Hz}$, $\left.\mathrm{H}-1^{\prime} \mathrm{a}\right), 2.22$ (1H, dd, $\left.J=14.6,7.6 \mathrm{~Hz}, \mathrm{H}-1^{\prime} \mathrm{b}\right), 2.05-1.65(4 \mathrm{H}, \mathrm{m}$, $\mathrm{H}-4^{\prime}$ and $\left.5^{\prime}\right), 1.57\left(3 \mathrm{H}, \mathrm{s}, \mathrm{H}-9^{\prime}\right), 1.72\left(3 \mathrm{H}, \mathrm{s}, \mathrm{H}-10^{\prime}\right)$, [the $\alpha-$ methoxy- $\alpha$-(trifluoromethyl)phenyl acetate portion exhibited $\delta 7.50$ and $\left.7.40\left(5 \mathrm{H}, \mathrm{m}, \mathrm{C}_{6} \mathrm{H}_{5}\right), 3.52\left(3 \mathrm{H}, \mathrm{s}, \mathrm{OCH}_{3}\right)\right]$.

2.4.2 Compound 2b. Colorless viscous liquid; ${ }^{1} \mathrm{H}$ NMR $\left(\mathrm{CDCl}_{3}, 400 \mathrm{MHz}\right) \delta 6.76(1 \mathrm{H}, \mathrm{dd}, J=10.3,3.5 \mathrm{~Hz}, \mathrm{H}-6), 6.19(1 \mathrm{H}$, brs, $J=10.3 \mathrm{~Hz}, \mathrm{H}-5), 5.37$ ( $1 \mathrm{H}$, ddd, $J=17.5,6.7,5.5 \mathrm{~Hz}, \mathrm{H}-6^{\prime}$ ), 5.05 ( $\left.1 \mathrm{H}, \mathrm{dt}, J=14.3,7.7,7.7 \mathrm{~Hz}, \mathrm{H}-2^{\prime}\right), 5.02$ (1H, brs, H-8'a), 4.98 (1H, brs, H-8'b), 4.87 (1H, dd, $J=3.3,1.2 \mathrm{~Hz}, \mathrm{H}-6 \mathrm{a}), 2.56$ $(1 \mathrm{H}, \mathrm{d}, J=16.7 \mathrm{~Hz}, \mathrm{H}-3 \alpha), 2.51(1 \mathrm{H}, J=16.7 \mathrm{~Hz}, \mathrm{H}-3 \beta), 2.46(1 \mathrm{H}$, $\mathrm{d}, J=17.3, \mathrm{~Hz}, \mathrm{H}-2 \alpha), 2.39(1 \mathrm{H}, \mathrm{d}, J=17.3, \mathrm{~Hz}, \mathrm{H}-2 \beta), 2.30(1 \mathrm{H}$, $\left.\mathrm{dd}, J=14.2,7.8 \mathrm{~Hz}, \mathrm{H}-1^{\prime} \mathrm{a}\right), 2.22\left(1 \mathrm{H}, \mathrm{dd}, J=14.2,7.6 \mathrm{~Hz}, \mathrm{H}-1^{\prime} \mathrm{b}\right)$, 2.09-1.90 (2H, m, H-4'), 1.90-165 (2H, m, H-5'), $1.72(3 \mathrm{H}, \mathrm{s}, \mathrm{H}-$ $10^{\prime}$ ), $1.59\left(3 \mathrm{H}, \mathrm{s}, \mathrm{H}-9^{\prime}\right)$, [the $\alpha$-methoxy- $\alpha$-(trifluoromethyl)phenyl acetate portion exhibited $\delta 7.50$ and $7.41\left(5 \mathrm{H}, \mathrm{m}, \mathrm{C}_{6} \mathrm{H}_{5}\right), 3.52$ $\left.\left(3 \mathrm{H}, \mathrm{s}, \mathrm{OCH}_{3}\right)\right]$.

\subsection{Preparation of the $(R)$-and $(S)$ - $\alpha$-methoxy- $\alpha$ - (trifluoromethyl) phenyl acetate of (5)}

The esterification of $\mathbf{5}$ was carried out using the procedure described for the preparation of $\mathbf{2 a}$ and $\mathbf{2} \mathbf{b}$ to yield $(S)$-ester (5a, $7 \mathrm{mg},(50 \%))$ and $(R)$-ester (5b, $12 \mathrm{mg},(71 \%))$.

2.5.1 Compound 5a. Colorless viscous liquid; ${ }^{1} \mathrm{H}$ NMR $\left(\mathrm{CDCl}_{3}, 400 \mathrm{MHz}\right) \delta 6.03(1 \mathrm{H}, \mathrm{d}, J=11.4 \mathrm{~Hz}, \mathrm{H}-5), 5.97(1 \mathrm{H}, \mathrm{dd}, J$ $=11.4,1.7 \mathrm{~Hz}, \mathrm{H}-6), 5.65(1 \mathrm{H}, \mathrm{t}, J=5.8 \mathrm{~Hz}, \mathrm{H}-4), 5.07(1 \mathrm{H}, \mathrm{t}, J=$ $\left.7.6 \mathrm{~Hz}, \mathrm{H}-2^{\prime}\right), 5.01\left(1 \mathrm{H}, \mathrm{t}, J=6.6 \mathrm{~Hz}, \mathrm{H}-6^{\prime}\right), 4.64$ (1H, brs, H-6a), $2.41(1 \mathrm{H}, J=17.4 \mathrm{~Hz}, \mathrm{H}-2 \alpha), 2.35(1 \mathrm{H}, J=17.4 \mathrm{~Hz}, \mathrm{H}-2 \beta), 2.21$ $\left(1 \mathrm{H}, \mathrm{dd}, J=14.4,8.0 \mathrm{~Hz}, \mathrm{H}-1^{\prime} \mathrm{a}\right), 2.18(1 \mathrm{H}, \mathrm{dd}, J=13.9,4.8 \mathrm{~Hz}, \mathrm{H}-$ $3 \beta), 2.14\left(1 \mathrm{H}, \mathrm{dd}, J=14.4,7.4 \mathrm{~Hz}, \mathrm{H}^{-1} \mathrm{I}^{\prime} \mathrm{b}\right), 2.08-1.98$ (4H, m, H-4' and $\left.5^{\prime}\right), 1.71(1 \mathrm{H}, \mathrm{dd}, J=13.9,7.9 \mathrm{~Hz}, \mathrm{H}-3 \alpha), 1.65\left(3 \mathrm{H}, \mathrm{s}, \mathrm{H}-8^{\prime}\right)$, $1.58\left(3 \mathrm{H}, \mathrm{s}, \mathrm{H}-10^{\prime}\right), 1.55\left(3 \mathrm{H}, \mathrm{s}, \mathrm{H}-9^{\prime}\right)$, [the $\alpha$-methoxy- $\alpha$-(trifluoromethyl)phenyl acetate portion exhibited $\delta 7.50$ and 7.41 $\left.\left(5 \mathrm{H}, \mathrm{m}, \mathrm{C}_{6} \mathrm{H}_{5}\right), 3.55\left(3 \mathrm{H}, \mathrm{s}, \mathrm{OCH}_{3}\right)\right]$.

2.5.2 Compound 5b. Colorless viscous liquid; ${ }^{1} \mathrm{H}$ NMR $\left(\mathrm{CDCl}_{3}, 400 \mathrm{MHz}\right) \delta 5.95(1 \mathrm{H}, \mathrm{d}, J=11.0 \mathrm{~Hz}, \mathrm{H}-5), 5.92(1 \mathrm{H}, \mathrm{d}, J=$ $11.0 \mathrm{~Hz}, \mathrm{H}-6), 5.66(1 \mathrm{H}, \mathrm{t}, J=6.8 \mathrm{~Hz}, \mathrm{H}-4), 5.12(1 \mathrm{H}, \mathrm{t}, J=7.7 \mathrm{~Hz}$, $\left.\mathrm{H}-2^{\prime}\right), 5.03\left(1 \mathrm{H}, \mathrm{t}, J=6.6 \mathrm{~Hz}, \mathrm{H}-6^{\prime}\right), 4.63(1 \mathrm{H}, \mathrm{brs}, \mathrm{H}-6 \mathrm{a}), 2.44(1 \mathrm{H}$, $J=17.4 \mathrm{~Hz}, \mathrm{H}-2 \alpha), 2.35(1 \mathrm{H}, J=17.4 \mathrm{~Hz}, \mathrm{H}-2 \beta), 2.24(1 \mathrm{H}, \mathrm{dd}, J=$ 14.6, $\left.5.5 \mathrm{~Hz}, \mathrm{H}-1^{\prime} \mathrm{a}\right), 2.21$ (1H, dd, $\left.J=14.0,4.3 \mathrm{~Hz}, \mathrm{H}-3 \beta\right), 2.18$ $\left(1 \mathrm{H}, \mathrm{dd}, J=14.6,7.6 \mathrm{~Hz}, \mathrm{H}-1^{\prime} \mathrm{b}\right), 2.09-2.02\left(4 \mathrm{H}, \mathrm{m}, \mathrm{H}-4^{\prime}\right.$ and $\left.5^{\prime}\right)$, $1.82(1 \mathrm{H}, \mathrm{dd}, J=14.0,8.2 \mathrm{~Hz}, \mathrm{H}-3 \alpha), 1.65\left(3 \mathrm{H}, \mathrm{s}, \mathrm{H}-8^{\prime}\right), 1.59$ $\left(3 \mathrm{H}, \mathrm{s}, \mathrm{H}-10^{\prime}\right), 1.57\left(3 \mathrm{H}, \mathrm{s}, \mathrm{H}-9^{\prime}\right)$, [the $\alpha$-methoxy- $\alpha$-(trifluoromethyl)phenyl acetate portion exhibited $\delta 7.50$ and 7.41 $\left.\left(5 \mathrm{H}, \mathrm{m}, \mathrm{C}_{6} \mathrm{H}_{5}\right), 3.54\left(3 \mathrm{H}, \mathrm{s}, \mathrm{OCH}_{3}\right)\right]$.

\subsection{X-ray crystallographic analyses of 10 and 11}

The reflection data were collected on a Bruker D8 Quest PHOTON100 CMOS detector with graphite-monochromated MoK $\alpha$ radiation using the APEX2 program. ${ }^{23}$ Raw data frame integration was performed with SAINT, ${ }^{23}$ which also applied correction for Lorentz and polarization effects. An empirical absorption correction using the SADABS program ${ }^{23}$ was applied. The structure was solved by direct methods and refined by fullmatrix least-squares method on $F^{2}$ with anisotropic thermal parameters for all non-hydrogen atoms using the SHELXTL software package. ${ }^{24}$ All hydrogen atoms were placed in calculated positions and refined isotropically. Crystallographic data of $\mathbf{1 0}$ and $\mathbf{1 1}$ were deposited with the following Cambridge Crystallographic Data Centre codes: CCDC 1415288 and CCDC 1062125, respectively.

2.6.1 Crystal data of 10. $\mathrm{C}_{30} \mathrm{H}_{28} \mathrm{O}_{8}\left(M=516.52 \mathrm{~g} \mathrm{~mol}^{-1}\right)$ : orthorhombic, space group Pna2 1 (no. 33), $a=23.725$ (4) $\AA$, $b=$ 5.5211(10) ̊, $c=39.408(7) \AA, V=5162.0(16) \AA^{3}, Z=8, T=$ $293(2) \mathrm{K}, \mu(\mathrm{MoK} \alpha)=0.096 \mathrm{~mm}^{-1}, D_{\text {calc }}=1.329 \mathrm{~g} \mathrm{~cm}^{-3}, 135470$ reflections measured $\left(6.204^{\circ} \leq 2 \Theta \leq 52.798^{\circ}\right), 10518$ unique $\left(R_{\text {int }}=0.0929, R_{\text {sigma }}=0.0445\right)$ which were used in all calculations. The final $R_{1}$ was $0.0508(I>2 \sigma(I))$ and $w R_{2}$ was 0.1225 (all data).

2.6.2 Crystal data of 11. $\mathrm{C}_{31} \mathrm{H}_{29} \mathrm{Cl}_{3} \mathrm{O}_{8}, \mathrm{MW}=635.89 \mathrm{~g}$ $\mathrm{mol}^{-1}$, triclinic, space group $P \overline{1}, a=11.6555(5) \AA ⿻$, $b=12.1165(5)$ $\AA, c=12.1203(5) \AA, \alpha=113.9720(10), \beta=102.4530(10), \gamma=$ 92.9570(10), $V=1508.54(11) \AA^{3}, Z=2, T=293(2) \mathrm{K}, \mu(\mathrm{MoK} \alpha)=$ $0.354 \mathrm{~mm}^{-1}, D_{\text {calc }}=1.400 \mathrm{~g} \mathrm{~cm}^{-3}, 61914$ reflections measured, 7472 unique $\left(R_{\text {int }}=0.0262\right)$ which were used in all calculations. The final $R_{1}$ was $0.0642(I>2 \sigma(I))$ and $\mathrm{w} R_{2}$ was 0.2211 (all data).

\subsection{Antimalarial assay}

Antimalarial activity was performed against P. falciparum (K1, multidrug resistant strain, see ESI $\dagger$ ), using the method of Trager and Jensen. ${ }^{25}$ Quantitative assessment of malarial activity in vitro was determined by means of the microculture radioisotope technique based upon the method described by Desjardins et $a .^{26}$ The inhibitory concentration $\left(\mathrm{IC}_{50}\right)$ represents the concentration that causes $50 \%$ reduction in parasite growth as indicated by the in vitro uptake of $\left[{ }^{3} \mathrm{H}\right]$-hypoxanthine by $P$. falciparum. The standard compound was dihydroartemisinin.

\subsection{Antimycobacterial assay}

Antimycobacterial activity was performed against $M$. tuberculosis H37Ra (purchased from ATCC) using the MicroplateAlamar Blue Assay (MABA). ${ }^{27}$ The standard drug streptomycin was used as reference substance.

\subsection{Cytotoxicity assay}

Cytotoxicity assays against human epidermoid carcinoma (KB), human breast cancer (MCF7), and human small cell lung cancer (NCI-H187) cell lines human breast adenocarcinoma Resazurin microplate assay described by O'Brien and co-workers. ${ }^{28}$ The reference substances were ellipticine and doxorubicin. Cytotoxicity test against primate cell line (Vero) was performed using 
the green fluorescent protein detection method described by Hunt and co-workers. ${ }^{29}$ The reference substances used were ellipticine and doxorubicin. All cells were purchased from ATCC.

\section{Results and discussion}

Chromatographic fractionation of $n$-hexane and EtOAc extracts yielded eight new bicyclic lactones, velutinones $\mathrm{A}-\mathrm{H}$ (1-8), three cyclobutane dimers, velutinindimers A-C (9-11), and four known compounds (12-15), kawapyrone, yangonin (12), ${ }^{30}$ three flavonoids, sakuranetin (13), ${ }^{31}$ 7-O-methyleriodictyol $(\mathbf{1 4})^{32}$ and rhamnetin $(\mathbf{1 5}),{ }^{33}$ and an acetogenin, cananginone $\mathrm{H}(\mathbf{1 6})^{20,21}$ (Fig. 1).

The IR spectra of 1-4 showed absorption bands of a $\gamma$ lactone moiety at (1789-1771 $\left.\mathrm{cm}^{-1}\right)$ and a conjugated carbonyl functionality (1685-1862 $\mathrm{cm}^{-1}$ ) similar to the absorption bands of a synthetic bicyclic cyclohexenone. ${ }^{34,35}$

Compound 1 possessed the molecular formula $\mathrm{C}_{18} \mathrm{H}_{24} \mathrm{O}_{3}$ based on the ${ }^{13} \mathrm{C}$ NMR and HRESITOFMS $(\mathrm{m} / \mathrm{z} 311.1611[\mathrm{M}+$ $\mathrm{Na}]^{+}$) data, indicating seven indices of hydrogen deficiency. The ${ }^{1} \mathrm{H}$ NMR data (Table 1$)$ had resonances at $\delta 2.48(\mathrm{~d}, J=17.4 \mathrm{~Hz}$, H-2 $\alpha$ ), 2.39 (d, $17.4 \mathrm{~Hz}, \mathrm{H}-2 \beta$ ), 2.52 (s, 2H, H-3), 6.17 (dd, $J=$ 10.3, 1.2 Hz, H-5), 6.75 (dd, $J=10.3,3.3 \mathrm{~Hz}, \mathrm{H}-6$ ), and 4.87 (dd, $J$ $=3.3,1.2 \mathrm{~Hz}, \mathrm{H}-6 \mathrm{a}$ ). The ${ }^{13} \mathrm{C}$ NMR data (Table 1), DEPT, and HMQC experiments indicated seven resonances which were associated with an $\alpha, \beta$-unsaturated carbonyl ( $\delta$ 196.2/C-4), a lactone carbonyl $(\delta 174.2 / \mathrm{C}-1)$, two olefinic $(\delta 131.2 / \mathrm{C}-5$ and 141.4/C-6), two methylene ( $\delta 38.9 / \mathrm{C}-2$ and 42.6/C-3), one methine $(\delta 77.8 / \mathrm{C}-6 \mathrm{a})$, and one quaternary $(\delta 44.7 / \mathrm{C}-2 \mathrm{a})$ carbons. Interpretation of the COSY and HMBC correlations (Fig. 2) indicated that $\mathbf{1}$ has a core structure of a five-membered lactone ring fused to an $\alpha, \beta$-unsaturated cyclohexanone ring. This arrangement is similar to that of a compound isolated from the fruit kernels of Otoba parvifolia ${ }^{35,36}$ and from a total synthesis of its core structure, ${ }^{34}$ except for the side chain at C-2a which was replaced by a geranyl moiety in $\mathbf{1}$. This geranyl side chain $\left(\mathrm{C}_{10} \mathrm{H}_{17}\right)$ was evident from the ${ }^{1} \mathrm{H}$ and ${ }^{13} \mathrm{C}$ NMR spectroscopic data (Table 1). The COSY spectrum showed the connectivity of the geranyl side chain by correlations between $\mathrm{H}-1^{\prime}$ and $\mathrm{H}-2^{\prime}$, and amongst $\mathrm{H}-4^{\prime}, \mathrm{H}-5^{\prime}$ and $\mathrm{H}-6^{\prime}$. The HMBC spectrum exhibited correlations of $\mathrm{H}-1^{\prime}$ to $\mathrm{C}-2, \mathrm{C}-3, \mathrm{C}-2 \mathrm{a}, \mathrm{C}-6 \mathrm{a}, \mathrm{C}-2^{\prime}$ and $\mathrm{C}-3^{\prime} ; \mathrm{H}-2^{\prime}$ to $\mathrm{C}-2 \mathrm{a}, \mathrm{C}-1^{\prime}, \mathrm{C}-3^{\prime}, \mathrm{C}-4^{\prime}$, and $\mathrm{C}-10^{\prime} ; \mathrm{H}-4^{\prime}$ to $\mathrm{C}-2^{\prime}, \mathrm{C}-3^{\prime}, \mathrm{C}-5^{\prime}, \mathrm{C}-6^{\prime}$ and $\mathrm{C}-10^{\prime} ; \mathrm{H}-6^{\prime}$ to $\mathrm{C}-4^{\prime}, \mathrm{C}-5^{\prime}, \mathrm{C}-8^{\prime}$, and $\mathrm{C}-9^{\prime}$ indicating that the geranyl group was linked to the stereogenic quaternary carbon C-2a (Fig. 2). The relative configuration at C-2a and C-6a was

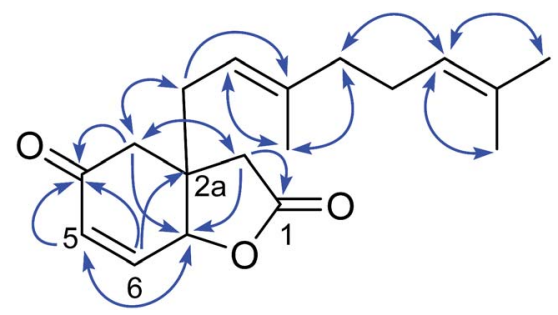

Fig. 2 Selected HMBC correlations of 1. established as syn from the NOESY correlation of $\mathrm{H}-6 \mathrm{a}$ and $\mathrm{H}-\mathbf{1}^{\prime}$. Based on the above evidence, the structure of $\mathbf{1}$, velutinone A, was defined as shown in Fig. 1.

Compound 2 had the molecular formula $\mathrm{C}_{18} \mathrm{H}_{24} \mathrm{O}_{4}$ derived from the ${ }^{13} \mathrm{C}$ NMR and HRESITOFMS $\left(m / z\right.$ 305.1740 $\left.[\mathrm{M}+\mathrm{H}]^{+}\right)$ data, demonstrating the same index of hydrogen deficiency, but having one additional oxygen atom compared to 1 . The ${ }^{1} \mathrm{H}$ and ${ }^{13} \mathrm{C}$ NMR spectroscopic data of 2 (Table 1 ) were similar to those of 1 , except for the geranyl side chain being oxidized at C- $6^{\prime}$ and having a $7^{\prime}, 8^{\prime}$ terminal double bond. The NMR spectroscopic data displayed resonances for an olefinic methylene protons at $\delta_{\mathrm{H}} 4.82\left(\mathrm{t}, J=1.4 \mathrm{~Hz}, 4.91\right.$ (brs), $\left.\mathrm{H}-8^{\prime}\right) / \delta_{\mathrm{C}} 111.1$ and an oxymethine at $\delta_{\mathrm{H}} 4.00\left(\mathrm{t}, J=6.3 \mathrm{~Hz}, \mathrm{H}-6^{\prime}\right) / \delta_{\mathrm{C}}$ 75.3. The HMBC correlations of $\mathrm{H}-5^{\prime}$ to $\mathrm{C}-3^{\prime}, \mathrm{C}-4^{\prime}, \mathrm{C}-6^{\prime}$, and C-7' ${ }^{\prime}$ H- $6^{\prime}$ to C- $4^{\prime}, \mathrm{C}-5^{\prime}$, $\mathrm{C}-7^{\prime}, \mathrm{C}-8^{\prime}$, and $\mathrm{C}-9^{\prime}$; and $\mathrm{H}-8^{\prime}$ to $\mathrm{C}-6^{\prime}, \mathrm{C}-7^{\prime}$, and $\mathrm{C}-9^{\prime}$ confirmed the position of the terminal olefinic moiety and the hydroxy group in the side chain. The assignment of the $\left(6^{\prime} S\right)$ absolute configuration was done via the modified Mosher's ester method ${ }^{22}$ (Fig. 3). Therefore, the structure of compound 2, velutinone B, was defined as shown in Fig. 1.

Compound 3 had the molecular formula $\mathrm{C}_{18} \mathrm{H}_{24} \mathrm{O}_{4}$, deduced from ${ }^{13} \mathrm{C}$ NMR and HRESITOFMS $\left(\mathrm{m} / z\right.$ 327.1543 $\left.[\mathrm{M}+\mathrm{Na}]^{+}\right)$data, implying the same index of hydrogen deficiency, but having one additional oxygen atom compared to 1 . The ${ }^{1} \mathrm{H}$ and ${ }^{13} \mathrm{C}$ NMR spectroscopic data of $\mathbf{3}$ (Table 1 ) were similar to those of $\mathbf{1}$, except for the appearance of the olefinic protons at $\delta_{\mathrm{H}} 5.51(\mathrm{dt}, J$ $\left.=15.6,6.6 \mathrm{~Hz}, \mathrm{H}-5^{\prime}\right)$ and $\delta_{\mathrm{H}} 5.60\left(\mathrm{~d}, J=15.6 \mathrm{~Hz}, \mathrm{H}-6^{\prime}\right)$, and one additional oxygenated carbon signal at $\delta_{\mathrm{C}} 70.4\left(\mathrm{C}-7^{\prime}\right)$. The position of the hydroxy group at $\mathrm{C}-7^{\prime}$ on the side chain was confirmed by the HMBC correlations of $\mathrm{H}-5^{\prime}$ to $\mathrm{C}-3^{\prime}, \mathrm{C}-4^{\prime}, \mathrm{C}-6^{\prime}$, and $\mathrm{C}-7^{\prime}$; and $\mathrm{H}-6^{\prime}$ to $\mathrm{C}-4^{\prime}, \mathrm{C}-5^{\prime}, \mathrm{C}-7^{\prime}$ and $\mathrm{C}-8^{\prime} / \mathrm{C}-9^{\prime}$. Therefore, the structure of compound 3, velutinone $\mathrm{C}$, was defined as shown (Fig. 1).

Compound 4 had the molecular formula $\mathrm{C}_{18} \mathrm{H}_{24} \mathrm{O}_{5}$ derived from the ${ }^{13} \mathrm{C}$ NMR and HRESITOFMS $\left(m / z\right.$ 343.1472 $\left.[\mathrm{M}+\mathrm{Na}]^{+}\right)$ data, demonstrating the same index of hydrogen deficiency, but having one additional oxygen atom compared to 3 . The NMR spectroscopic data of 4 (Table 1 ) was similar to that of 3 , except for the resonance of a C-7' hydroxy group. The ${ }^{13} \mathrm{C}$ NMR spectrum revealed the unusual downfield oxygenated carbon signal at $\delta_{\mathrm{C}} 81.8$ for $\mathrm{C}^{-7^{\prime}}$, suggesting the presence of a hydroperoxy group. ${ }^{37}$ Thus the structure of compound $\mathbf{4}$, velutinone $\mathrm{D}$, was determined as shown (Fig. 1).

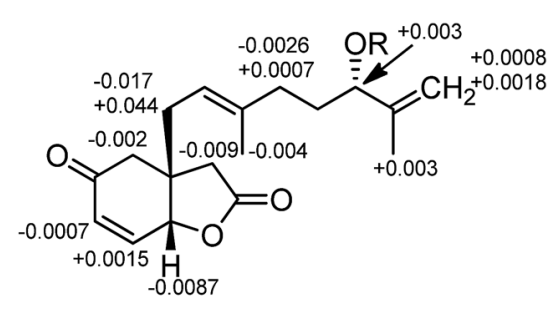

2a: $\mathrm{R}=(S)-\mathrm{MTPA}$

2b: $\mathrm{R}=(R)-\mathrm{MTPA}$

Fig. $3 \Delta \delta$ values ( $\Delta \delta=\delta_{S}-\delta_{R}$ in ppm) obtained for MTPA esters 2a and $2 b$. 
Compound 5 had the molecular formula $\mathrm{C}_{18} \mathrm{H}_{26} \mathrm{O}_{3}$ derived from the ${ }^{13} \mathrm{C}$ NMR and HRESITOFMS $\left(m / z\right.$ 291.1935 $\left.[\mathrm{M}+\mathrm{Na}]^{+}\right)$ data, implying six indices of hydrogen deficiency. The IR spectrum showed hydroxy $\left(3442 \mathrm{~cm}^{-1}\right)$ and $\gamma$-lactone carbonyl (1771 $\mathrm{cm}^{-1}$ ) functionalities. The NMR data of 5 (Table 2) corresponded to those of $\mathbf{1}$, except that the ketone carbonyl resonance for $\mathrm{C}-4$ was replaced by a resonance for an oxymethine group at $\delta_{\mathrm{H}} 4.31(\mathrm{~m}) / \delta_{\mathrm{C}} 63.7$. The assignment of $(4 S)$ absolute configuration was done via the modified Mosher's ester $\operatorname{method}^{22}$ (Fig. 4). The configurations of $(2 \mathrm{a} R$ and $6 \mathrm{a} R)$ were assigned by NOESY and a molecular modeling study. NOESY correlations were observed between $\mathrm{H}-4$ and $\mathrm{H}-2 \alpha, \mathrm{H}-6 \mathrm{a}$ and $\mathrm{H}-$ $1^{\prime} \mathrm{a}, \mathrm{H}-6 \mathrm{a}$ and $\mathrm{H}-3 \beta, \mathrm{H}-1^{\prime} \mathrm{b}$ and $\mathrm{H}-2 \beta$, and $\mathrm{H}-3 \alpha$ and $\mathrm{H}-2^{\prime}$ (Fig. 5). Therefore, the structure of compound 5 , velutinone $\mathrm{E}$, was established as shown (Fig. 1).

Compound 6 had the molecular formula $\mathrm{C}_{18} \mathrm{H}_{24} \mathrm{O}_{4}$ derived from the ${ }^{13} \mathrm{C}$ NMR and HRESITOFMS $\left(m / z\right.$ 327.1562 $\left.[\mathrm{M}+\mathrm{Na}]^{+}\right)$ data, implying the same index of hydrogen deficiency, but having one more oxygen atom than 1 . The IR spectrum showed bands for $\gamma$-lactone $\left(1789 \mathrm{~cm}^{-1}\right.$ and a cyclohexanone (1719 $\mathrm{cm}^{-1}$ ) groups. The NMR data of $\mathbf{6}$ (Table 2) was similar to that of 1, except for the resonances of the C-5/6 double bond which were replaced by those of an epoxide moiety $\left[\delta_{\mathrm{H}} 3.65(\mathrm{~d}, J=\right.$ $3.6 \mathrm{~Hz}, \mathrm{H}-5) / \delta_{\mathrm{C}} 58.6$ and $\left.\left.\delta_{\mathrm{H}} 3.37(\mathrm{~d}, J=3.6 \mathrm{~Hz}, \mathrm{H}-6) / \delta_{\mathrm{C}} 54.9\right)\right]$. The relative configuration of the epoxide was assigned by the NOESY correlations between H-6 and H-6a. Hence, the structure of compound 6, velutinone F, was defined as shown (Fig. 1).

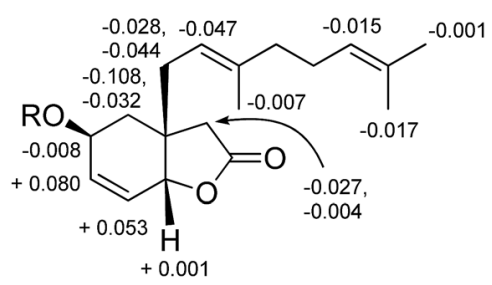

$$
\begin{aligned}
& \text { 5a, } \mathrm{R}=(S)-\mathrm{MTPA} \\
& \text { 5b, } \mathrm{R}=(R)-\mathrm{MTPA}
\end{aligned}
$$

Fig. $4 \Delta \delta$ values ( $\Delta \delta=\delta_{S}-\delta_{R}$ in ppm) obtained for MTPA esters 5 a and $5 b$.

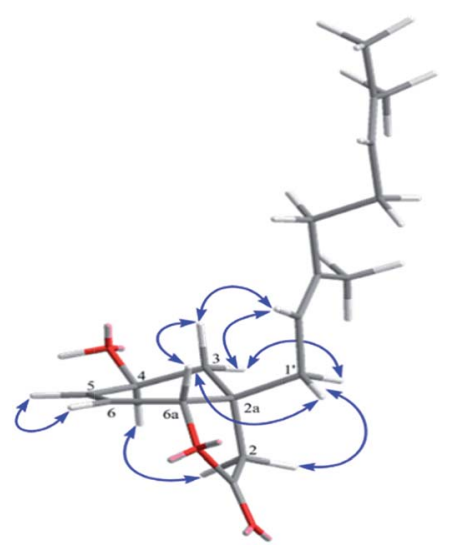

Fig. 5 Key NOESY correlations of 5, energy minimized using MM2.
Compound 7 had the molecular formula $\mathrm{C}_{18} \mathrm{H}_{24} \mathrm{O}_{5}$, established from the ${ }^{13} \mathrm{C}$ NMR and HRESITOFMS $(\mathrm{m} / \mathrm{z} 321.1690[\mathrm{M}+$ $\mathrm{Na}]^{+}$) data, having the same index of hydrogen deficiency as in 2 . The IR spectrum displayed an extra hydroxyl band at (3481 $\mathrm{cm}^{-1}$ ) which differed from that of 6 . The NMR data of 7 (Table 2) corresponded to that of 2, except for the resonances of a C-5/6 double bond, which was replaced by resonances for an epoxide moiety $\left[\delta_{\mathrm{H}} 3.62(\mathrm{~d}, J=3.6 \mathrm{~Hz}, \mathrm{H}-5) / \delta_{\mathrm{C}} 58.5\right.$ and $\delta_{\mathrm{H}} 3.34$ $\left.(\mathrm{d}, J=3.6 \mathrm{~Hz}, \mathrm{H}-6) / \delta_{\mathrm{C}} 54.8\right]$ as in that of 6 . The $6^{\prime} S$ configuration was assigned by comparison of the NMR data to that of 2 . Thus the structure of 7, velutinone $\mathrm{G}$, was defined as shown (Fig. 1).

Compound 8 possessed the molecular formula $\mathrm{C}_{18} \mathrm{H}_{24} \mathrm{O}_{5}$ from the ${ }^{13} \mathrm{C}$ NMR and HRESITOFMS $\left.(m / z) 321.1690[\mathrm{M}+\mathrm{H}]^{+}\right)$ data, having seven indices of hydrogen deficiency as in $\mathbf{6}$, but having one additional oxygen. The IR spectrum was also similar to that of $\mathbf{6}$. The NMR data of $\mathbf{8}$ (Table 2) corresponded to that of 6, except for the resonances for a $\mathrm{C}-6^{\prime} / 7^{\prime}$ double bond which were replaced by resonances for an epoxide $\left[\delta_{\mathrm{H}} 3.65(\mathrm{t}, J=\right.$ $6.7 \mathrm{~Hz}, \mathrm{H}-6^{\prime} / \delta_{\mathrm{C}} 63.7$ and $\left.\left.\delta_{\mathrm{C}} 58.2, \mathrm{C}-7^{\prime}\right)\right]$ at this position. The configuration at C-6 $6^{\prime}$ of $\mathbf{8}$ was proposed to be $S$, based on its ring opening to give 7 . Hence, structure of $\mathbf{8}$, velutinone $\mathrm{H}$, was designated as shown in Fig. 1.

There are only two closely related bicyclic lactones, panamonons $\mathrm{A}$ and $\mathrm{B},{ }^{38}$ which have NMR data similar to those of compounds 1-8. However, the relative configuration at the ring junction (C-2a and C-6a) of 1-8 were assigned as $2 \mathrm{a} R$ and $6 \mathrm{a} R$ which different from panamonons $\mathrm{A}$ and $\mathrm{B}(2 \mathrm{a} R$ and $6 \mathrm{a} S) \cdot{ }^{38}$ The ECD spectra of compounds 1-4, containing an $\alpha, \beta$-unsaturated ketone, and compound 5, showed negative Cotton effects in the range of 202-215 $\mathrm{nm}$. While, compounds 6-8 contained a saturated ketone, exhibited positive Cotton effects in the range of 205-215 nm (Fig. 6).

To confirm the natural occurrence of 1-8, the isolates velutinone A (1) with a geranyl side chain and velutinone $\mathrm{H}(\mathbf{8})$ with two epoxide rings were stirred with or without silica gel in EtOAc and $\mathrm{MeOH}$ for 4 days following the conditions of the separation process. No change on TLC was observed. We conclude that isolates 1-8 are natural occurring products not artefacts.

The putative biosynthetic pathway towards compounds 1$\mathbf{8}$ is shown in Fig. 7. The precursor, homogentisic acid, ${ }^{35,36}$ could be prenylated by geranyl diphosphate to form intermediate $\mathbf{A}^{38}$ which may be reduced and lactonized to form compound $\mathbf{1}$. Reduction of $\mathbf{1}$ would produce 5 , while epoxidation of 1 would afford 6 or intermediate B. Oxidation of 5 would give $\mathbf{4}$ which could be reduced to give $\mathbf{3}$ or $\mathbf{B}$, the latter via intermediate $\mathbf{C}$. Protonation and deprotonation of $\mathbf{B}$ may give 2 and 3. The hydroxy group of 3 could be oxidized to give 4. Compound $\mathbf{8}$ could be derived from $\mathbf{6}$ or $\mathbf{B}$ via an oxidation reaction. Further protonation and deprotonation of $\mathbf{8}$ would give 7.

Compound 9 showed an $[\mathrm{M}+\mathrm{Na}]^{+}$ion peak at $m / z 539.1651$ in its HRESITOFMS, which in conjunction with the ${ }^{13} \mathrm{C}$ NMR data indicated the molecular formula $\mathrm{C}_{30} \mathrm{H}_{28} \mathrm{O}_{8}$, requiring seventeen indices of hydrogen deficiency. The IR spectrum showed bands for unsaturated lactone $\left(1715 \mathrm{~cm}^{-1}\right)$ and aromatic $\left(1643 \mathrm{~cm}^{-1}\right)$ groups. The UV spectrum also indicated an aromatic moiety $(286 \mathrm{~nm})$. Since the NMR spectroscopic data 

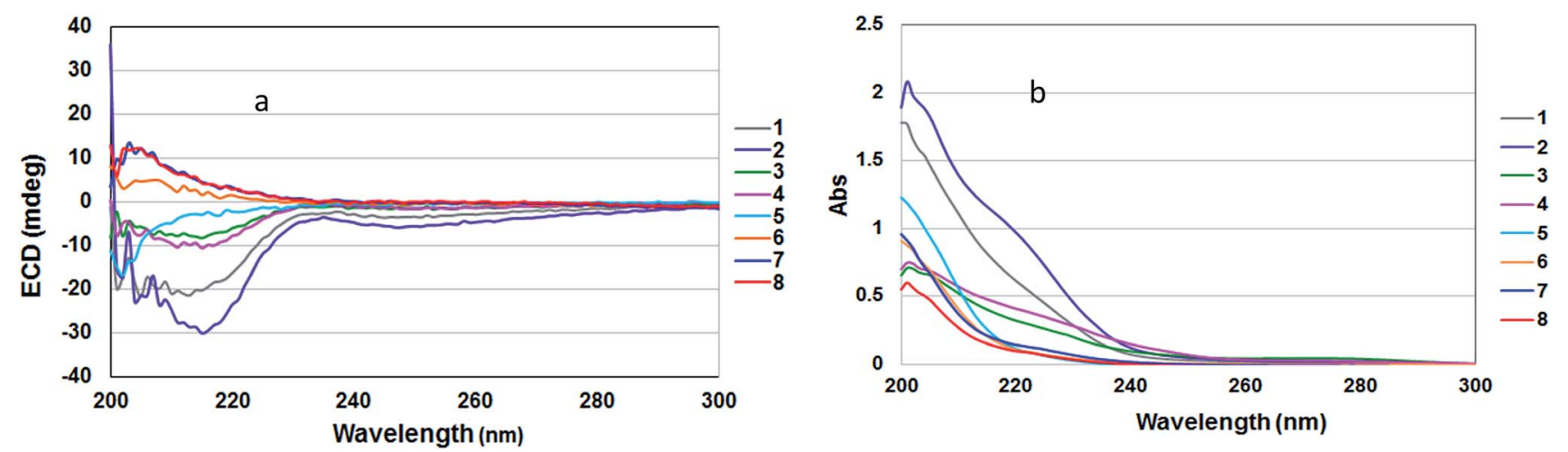

Fig. 6 ECD (a) and UV (b) spectra (in $\mathrm{MeOH}$ ) of compounds 1-8.

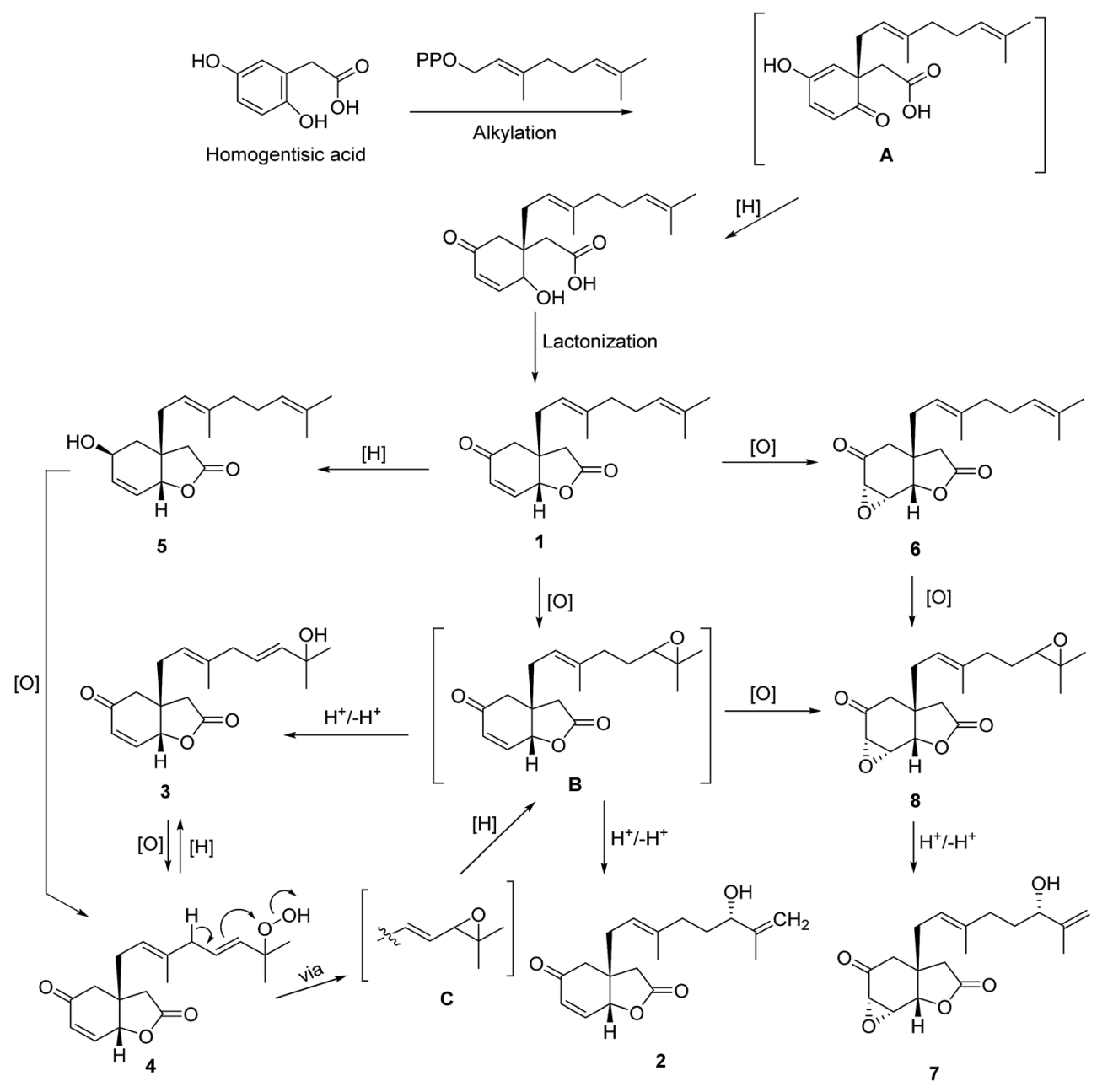

Fig. 7 Plausible biogenetic pathway of 1-8.

of 9 (Table 3) displayed half the number of resonance signals expected for 28 protons and 30 carbons, the structure should be a symmetrical dimer. These NMR resonances corresponded to those of the cyclobutane dimer, achyrodimer $\mathrm{A},{ }^{39}$ except for hydroxy groups at $\mathrm{C}-12$ and $\mathrm{C}-12^{\prime}$ of the aromatic rings which were replaced by methoxy groups. The ${ }^{1} \mathrm{H}$ NMR spectroscopic data (Table 3) showed resonances for the para-substituted benzene rings at $\delta 6.82$ and 7.19 (each $4 \mathrm{H}, \mathrm{d}, J=8.7 \mathrm{~Hz}$ ), four methines of the cyclobutyl ring at $\delta 4.16$ and 4.35 (each $2 \mathrm{H}$, dd, $J=7.6,10.0 \mathrm{~Hz}$ ), four olefinic protons for the two $\alpha$-pyrone moieities at $\delta 5.21$ and 5.71 (each $2 \mathrm{H}, \mathrm{d}, J=2.2 \mathrm{~Hz}$ ), and four methoxy groups at $\delta 3.75$ and 3.67 (each $6 \mathrm{H}, \mathrm{s}$ ). The ${ }^{13} \mathrm{C} \mathrm{NMR}$ data (Table 3) showed resonances for the para-disubstituted benzene rings at $\delta 129.4\left(\mathrm{C}-9,9^{\prime}\right), 128.5\left(\mathrm{C}-10,10^{\prime}\right.$ and $\left.\mathrm{C}-14,14^{\prime}\right)$, 

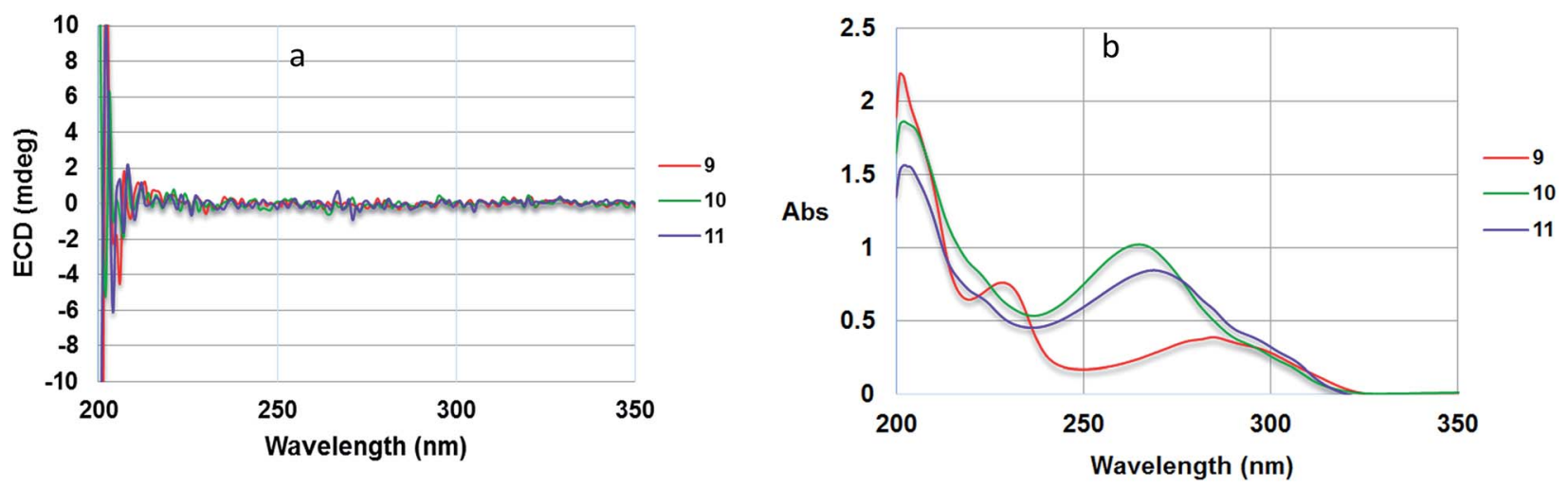

Fig. 8 ECD (a) and UV (b) spectra (in $\mathrm{MeOH}$ ) of compounds 9, 10 and 11.

and $113.9\left(\mathrm{C}-11,11^{\prime}\right)$, the cyclobutyl methines at $\delta 43.0\left(\mathrm{C}-7,7^{\prime}\right)$, and $45.5\left(\mathrm{C}-8,8^{\prime}\right)$ the $\alpha$-pyrone methines at $\delta 87.7\left(\mathrm{C}-3,3^{\prime}\right), 101.3$ $\left(\mathrm{C}-5,5^{\prime}\right)$ and methoxy groups at $\delta 55.7\left(4,4^{\prime}-\mathrm{OMe}\right)$ and $55.2(12$, $12^{\prime}$-OMe), and a carbonyl group at 162.9 (C-6, 6'). The correlations of $\mathrm{H}-7 / 7^{\prime}$ to $\mathrm{C}-5 / 5^{\prime}, \mathrm{C}-6 / 6^{\prime}, \mathrm{C}-8 / 8^{\prime}$, and C-9/9 ${ }^{\prime}$, and of $\mathrm{H}-8 / 8^{\prime}$ to $\mathrm{C}-6 / 6^{\prime}, \mathrm{C}-7 / 7^{\prime}, \mathrm{C}-10 / 10^{\prime}$, and $\mathrm{C}-14 / 14^{\prime}$ from the HMBC spectrum revealed the connection of a cyclobutane ring to an $\alpha$ pyrone ring, and benzene rings at $\mathrm{C}-7 / 7^{\prime}$ and $\mathrm{C}-8 / 8^{\prime}$, respectively. Resonances for two sets of methoxy protons at $\delta_{\mathrm{H}} 3.75$ and 3.67 showed correlations with $\mathrm{C}-4 / 4^{\prime}$ and $\mathrm{C}-12 / 12^{\prime}$, respectively, confirming the location of methoxy groups at C-4/4' and C-12/ $12^{\prime}$. The correlations between $\mathrm{H}-7\left(7^{\prime}\right)$ and $\mathrm{H}-8\left(8^{\prime}\right)$ in the NOESY spectrum indicated the relative configuration on the cyclobutane ring as reported for achyrodimer $\mathrm{A}^{39}$ The specific rotation value of 9 was almost zero $\left[+0.08\right.$ (c $0.63, \mathrm{MeOH}-\mathrm{CHCl}_{3}$; $3: 1)$ ] which was also the same as that reported for a symmetric achyrodimer A. ${ }^{39}$ Moreover, the ECD spectrum of 9 showed no signal for a Cotton effect (Fig. 8). ${ }^{40}$ Based on this evidence the structure of compound $\mathbf{9}$ could contain a plane of symmetry. Hence, 9 was concluded to be a new symmetrical cyclobutane dimer of the isolated styrylpyrone, yangonin (12), and it was named velutinindimer $\mathrm{A}$.

Compound 10 possessed the molecular formula $\mathrm{C}_{30} \mathrm{H}_{28} \mathrm{O}_{8}$ from the ${ }^{13} \mathrm{C}$ NMR and HRESITOFMS $\left(m / z\right.$ 539.1666 $\left.[\mathrm{M}+\mathrm{Na}]^{+}\right)$ data, having the same index of hydrogen deficiency as 9 . The IR spectrum displayed bands for lactone $\left(1699 \mathrm{~cm}^{-1}\right)$ and aromatic $\left(1647 \mathrm{~cm}^{-1}\right.$ ) groups. The UV spectrum also supported an aromatic moiety (268 nm). The ${ }^{1} \mathrm{H}$ NMR data of 10 (Table 3) showed resonances for two para-disubstituted benzene rings at $\delta 6.85,7.16$, and 7.34 and 6.85 (each $2 \mathrm{H}, \mathrm{d}, J=8.7 \mathrm{~Hz}$ ), an $E$ double bond at $\delta 6.42$ and 6.86 (each $1 \mathrm{H}, \mathrm{d}, J=15.8 \mathrm{~Hz}$ ), $\alpha$ pyrone ring at $\delta 5.33$ and 5.89 (each $1 \mathrm{H}, \mathrm{d}, J=2.2 \mathrm{~Hz}, \mathrm{H}-3$ and $\mathrm{H}-$ 5 , respectively) and $5.29\left(\mathrm{~s}, \mathrm{H}-3^{\prime}\right)$, three methine protons at $\delta 3.55\left(\mathrm{~d}, J=9.9 \mathrm{~Hz}, \mathrm{H}-5^{\prime}\right), 4.26$ (dd, $\left.J=10.8,9.9 \mathrm{~Hz}, \mathrm{H}-8\right), 4.09$ (d, $J=10.8 \mathrm{~Hz}, \mathrm{H}-7)$, and four methoxy groups at $\delta 3.32,3.69$, 3.78, and 3.79. The ${ }^{13} \mathrm{C}$ NMR spectrum, DEPT and HMQC experiments of 10 showed 30 resonances, including two sets of $p$-disubstituted benzene rings, one $\alpha$-pyrone ring, one olefinic, one cyclobutane ring, and four methoxy carbons. The HMBC spectrum displayed ${ }^{3} J$ correlations of $\mathrm{H}-3$ to C-5; $\mathrm{H}-5$ to C-3, and C-7; H-7 to C-5, C-9, C-5' ${ }^{\prime}$ and C-7'; H-8 to C-6, C-10, C-14, C-4', and $\mathrm{C}-6^{\prime} ; \mathrm{H}-10,14$ to $\mathrm{C}-8$, and $\mathrm{C}-12 ; \mathrm{H}-11,13$ to $\mathrm{C}-9 ; \mathrm{H}-3^{\prime}$ to $\mathrm{C}-5^{\prime}$; $\mathrm{H}-5^{\prime}$ to $\mathrm{C}-7, \mathrm{C}-9, \mathrm{C}-3^{\prime}$ and $\mathrm{C}-7^{\prime} ; \mathrm{H}-7^{\prime}$ to $\mathrm{C}-7, \mathrm{C}-5^{\prime}$, and $\mathrm{C}-9^{\prime} ; \mathrm{H}-8^{\prime}$ to $\mathrm{C}-6^{\prime}, \mathrm{C}-10^{\prime}$, and $\mathrm{C}-14^{\prime} ; \mathrm{H}-10^{\prime}, 14^{\prime}$ to $\mathrm{C}-8^{\prime}$ and $\mathrm{C}-12^{\prime} ; \mathrm{H}-11^{\prime}, 13^{\prime}$ to $\mathrm{C}-$ $9^{\prime}$ and C-12'; 4-OMe to C-4; $12-\mathrm{OMe}$ to C-12; $4^{\prime}$-OMe to C- $4^{\prime}$; and $12^{\prime}$-OMe to $\mathrm{C}-12^{\prime}$ confirming the structure of 10 (Fig. 9). The NMR data of $\mathbf{1 0}$ was comparable to the cyclobutane dimer achyrodimer D, reported from the aerial parts of Achyrocline bogotensis. ${ }^{39}$ It was found that $\mathbf{1 0}$ was the methoxy derivative of achyrodimer D. The relative configuration of $\mathbf{1 0}$ was determined from the relatively large coupling constants $(9.9-10.8 \mathrm{~Hz})$ between $\mathrm{H}-7$ and $\mathrm{H}-8$, and $\mathrm{H}-8$ and $\mathrm{H}-5^{\prime}$, and the NOESY correlations between $\mathrm{H}-8$ and $\mathrm{H}-5^{\prime}, \mathrm{H}-7$ and $\mathrm{H}-14, \mathrm{H}-8$ and $\mathrm{H}-10, \mathrm{H}-5$ and $\mathrm{H}-7, \mathrm{H}-\mathrm{7}^{\prime}$ and $\mathrm{H}-10^{\prime}$, and $\mathrm{H}-8^{\prime}$ and $\mathrm{H}-14^{\prime}$. The magnitude of the coupling constant between $\mathrm{H}-7$ and $\mathrm{H}-8\left(J_{\text {trans }}=10.8 \mathrm{~Hz}\right)$, and

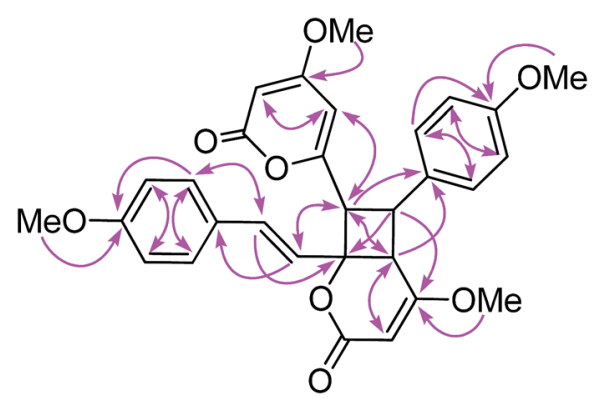

Fig. 9 Selected HMBC correlations of 10.

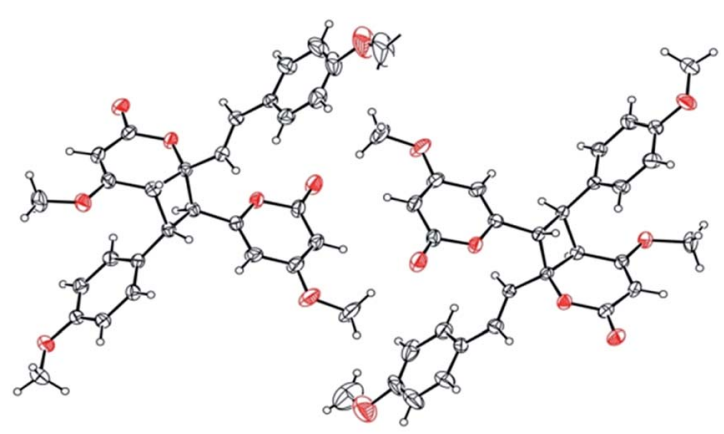

Fig. 10 ORTEP plot of the asymmetric units in 10. The thermal ellipsoids are shown at $40 \%$ probability. 


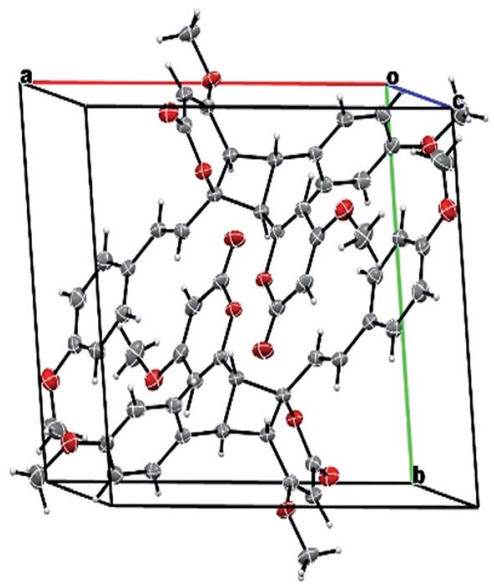

Fig. 11 Packing structure of 11 showing the inverted racemic mixture. The chloroform solvate molecule is omitted for clarity.

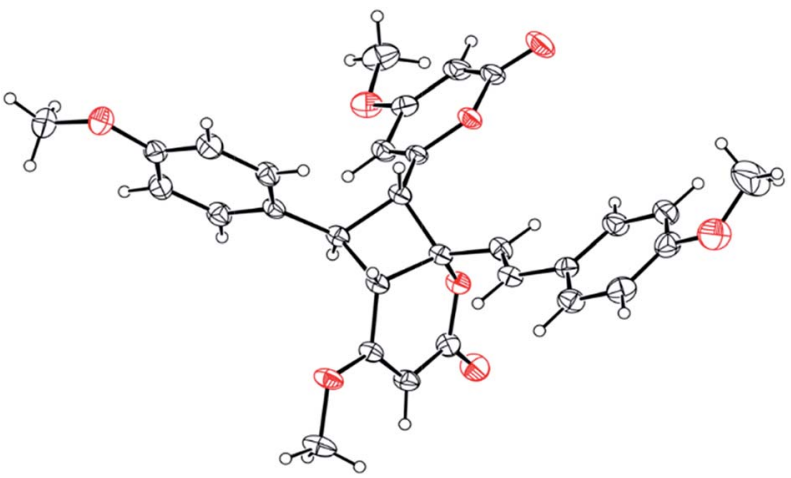

Fig. 12 ORTEP plot of the asymmetric unit in 11. The thermal ellipsoids are shown at $30 \%$ probability level. The chloroform solvate molecule is omitted for clarity.

$\mathrm{H}-8$ and $\mathrm{H}-5^{\prime}\left(\mathrm{J}_{\text {cis }}=9.9 \mathrm{~Hz}\right)$ could be correlated to the dihedral angle between those protons, which corresponded with the values from the Karplus equation for a four membered ring. ${ }^{41}$
The ECD measurement of $\mathbf{1 0}$ in $\mathrm{MeOH}$ showed no signal of the Cotton effect ${ }^{40}$ (Fig. 8), and also the specific rotation value of $\mathbf{1 0}$ was almost zero $(+0.08)$. These could suggest that compound $\mathbf{1 0}$ was a racemic mixture. Finally, the X-ray crystallographic analysis supported the structure of an isolated $\mathbf{1 0}$ containing asymmetric units of a racemic mixture (Fig. 10), and the one with the relative configuration $\left(5^{\prime} S, 6^{\prime} R, 7 S\right.$, and $\left.8 S\right)$ is shown in Fig. 1 . Thus, the structure of $\mathbf{1 0}$ was an unsymmetrical cyclobutane dimer of the isolated yangonin (12), and it was named velutinindimer B.

Compound 11 exhibited an $[\mathrm{M}+\mathrm{Na}]^{+}$peak at $m / z 539.1664$ in the positive HRESITOFMS corresponding to the molecular formula $\mathrm{C}_{30} \mathrm{H}_{28} \mathrm{O}_{8} \mathrm{Na}$, the same as that of 10 . The IR spectrum showed bands for a lactone moiety $\left(1708 \mathrm{~cm}^{-1}\right)$ and an aromatic ring $\left(1649 \mathrm{~cm}^{-1}\right)$. The UV spectrum indicated an aromatic moiety $(273 \mathrm{~nm})$. The NMR data (Table 3 ) and 2D NMR of 11 demonstrated a similar structure to a dimeric 10. Nevertheless, slight differences in chemical shifts around compounds $\mathbf{1 1}$ and 10 in the ${ }^{1} \mathrm{H}$ and ${ }^{13} \mathrm{C}$ NMR data at positions $7,8,9,5^{\prime}, 7^{\prime}$ and $8^{\prime}$ (Table 3) suggested different configurations at the cyclobutane ring between the two compounds. The large coupling constant of $\mathrm{H}-7$ and $\mathrm{H}-8\left(J_{\text {trans }}=10.3 \mathrm{~Hz}\right.$ ) and $\mathrm{H}-8$ and $\mathrm{H}-5^{\prime}$ (Jtrans $=9.7$ $\mathrm{Hz}$ ) could be explained in the same way as for $\mathbf{1 0}^{41}$ The NOESY spectrum displayed correlations of $\mathrm{H}-5^{\prime}$ and $\mathrm{H}-7, \mathrm{H}-7$ and $\mathrm{H}-14$, $\mathrm{H}-8$ and $\mathrm{H}-10, \mathrm{H}-\mathrm{-}^{\prime}$ and $\mathrm{H}-10^{\prime}$, and $\mathrm{H}-8^{\prime}$ and $\mathrm{H}-14^{\prime}$, indicating the relative configuration of 11. Compound $\mathbf{1 1}$ also showed no signal of the Cotton effect (Fig. 7) ${ }^{40}$ and also its specific rotation value was almost zero $(+0.3)$ suggesting that it should be a racemic mixture as compound $\mathbf{1 0}$. The X-ray crystallographic analysis confirmed that the isolated compound $\mathbf{1 1}$ was a racemic mixture as in $\mathbf{1 0}$, and the one with the relative configuration $5^{\prime} R, 6^{\prime} S, 7 R$, and $8 S$ is shown in Fig. 1, 11 and 12. From the above evidence, the structure of $\mathbf{1 1}$ was determined to be another new dimeric styrylpyrone and it was named velutinindimer $\mathrm{C}$.

To confirm the natural occurrence of styrylpyrone dimers, 911, the isolated yangonin (12), was stirred with silica gel in EtOAc and $\mathrm{MeOH}$ for a week, following the conditions for our

Table 4 Biological activities of the isolated compounds

\begin{tabular}{|c|c|c|c|c|c|c|}
\hline \multirow[b]{2}{*}{ Compound } & \multirow{2}{*}{$\frac{\text { Antimalarial }}{\left(\mathrm{IC}_{50}, \mu \mathrm{M}\right)}$} & \multirow{2}{*}{$\frac{\text { Anti-TB }}{(\mathrm{MIC}, \mu \mathrm{M})}$} & \multicolumn{4}{|c|}{ Cytotoxicity $\left(\mathrm{IC}_{50}, \mu \mathrm{M}\right)$} \\
\hline & & & $\mathrm{KB}^{a}$ & $\mathrm{MCF}^{b}$ & NCI-H1 $187^{c}$ & Vero cell ${ }^{d}$ \\
\hline 2 & 9.6 & 82.1 & 9.6 & 12.9 & 6.5 & 8.8 \\
\hline 3 & 10.0 & Inactive & 12.9 & 10.9 & 11.4 & 10.3 \\
\hline 4 & 9.6 & Inactive & 10.5 & 15.2 & 8.7 & 11.7 \\
\hline 8 & 7.3 & Inactive & 10.5 & 11.9 & 6.8 & 18.2 \\
\hline 9 & 6.4 & Inactive & Inactive & Inactive & Inactive & Inactive \\
\hline 10 & 5.4 & Inactive & Inactive & Inactive & Inactive & Inactive \\
\hline 11 & 5.8 & Inactive & Inactive & Inactive & Inactive & Inactive \\
\hline Dihydroartemisinin & 0.004 & & & & & \\
\hline Streptomycin & & $0.3-0.5$ & & & & \\
\hline
\end{tabular}


separation process. The formation of $\mathbf{9 ,} \mathbf{1 0}$ and $\mathbf{1 1}$ was not observed. It is worth noting that isolated dimers $\mathbf{1 0}$ and $\mathbf{1 1}$ were racemic mixtures occurring from asymmetrical $2+2$ cycloaddition of the isolated styrylpyrone, yangonin (12), while compound 9 has an axis of a symmetric dimer and so is not chiral.

The biological activities of the isolated compounds (purity $>$ 95\% from the NMR spectra) are shown in Table 4. Compounds 1 and 2, with MIC values of 43.4 and $82.1 \mu \mathrm{M}$ respectively, should be responsible for the antimycobacterial activity against $M$. tuberculosis exhibited in the primary screening. From this result, the $\alpha, \beta$-unsaturated carbonyl in the core structure plays an important role against TB, while the hydroxy or peroxide functionalities at $\mathrm{C}-6^{\prime}$ or $\mathrm{C}-\mathrm{7}^{\prime}$ reduce this activity. Since our previous work reported the antimalarial activity and cytotoxicity of compounds from M. velutina, ${ }^{20,21}$ the compounds isolated herein have been further evaluated for their activities. Compounds 2-4 and 7-11 displayed antimalarial activity toward $P$. falciparum with $\mathrm{IC}_{50}$ values in the range of 5.4-10.0 $\mu \mathrm{M}$. In addition, compounds 1-4 and 6-8 exhibited cytotoxicity against three cancer cell lines tested, with $\mathrm{IC}_{50}$ values in the range of 4.0-24.1 $\mu \mathrm{M}$. Among these, 1, 2 and 8 exhibited moderate cytotoxicity against NCI-H187 cell lines with $\mathrm{IC}_{50}$ values of 4.2, 6.5 and $6.8 \mu \mathrm{M}$, respectively, which were close to the standard drug, ellipticine (5.6 $\mu \mathrm{M})$. Compounds 1-4 and 6-8 exhibited cytotoxicity towards the Vero cell line with $\mathrm{IC}_{50}$ values in the range of 5.8-18.2 $\mu \mathrm{M}$. However, dimeric styrylpyrones 9-11 showed no cytotoxicity in the test.

\section{Conclusions}

Isolation of the leaves extracts of $M$. velutina yielded an unique class of eight bicyclic lactones with a $\mathrm{C}_{18}$ carbons architecture, named velutinones $\mathrm{A}-\mathrm{H}$ (1-8), three new dimeric styrylpyrones, velutinindimers A-C (9-11), five known compounds, the kawapyrone, yangonin (12), three flavonoids (13-15), and an acetogenin, cananginone $\mathrm{H}$ (16). Velutinindimers A-C (9-11) are dimmers occurring from symmetrical and asymmetrical $2+2$ cycloaddition of the isolated styrylpyrone, yangonin (12). The structures of velutinindimers B and C (10 and 11) were identified as mixtures which were confirmed by X-ray crystallographic, ECD and specific rotation analyses. Biological activity of the isolated compounds had been evaluated. Compounds 2-4 and 7-11showed antimalarial activity with $\mathrm{IC}_{50}$ values in the range of 5.4-10.0 $\mu \mathrm{M}$. Moreover, 1-4 and 6-8 displayed cytotoxicity against the KB, MCF7, and NCI-H187 cancer cell lines and Vero cell lines with $\mathrm{IC}_{50}$ values in the range of 4.0-24.1 $\mu \mathrm{M}$.

\section{Acknowledgements}

Financial support from the Thailand Research Fund and Khon Kaen University (Grant No. RTA 5980002) is gratefully acknowledged. We thanks the Center for Innovation in Chemistry (PERCH-CIC) for partial support. We are grateful to the Bioassay Research Facility of the BIOTEC, Thailand for biological activity assay.

\section{Notes and references}

$1 \mathrm{~W}$. Chuakul and N. Sornthornchareonon, Thai J. Phytopharm., 2003, 10, 25-32.

2 T. Smitinand, Thai Plant Names Revised Edition, Prachachon Co. Limited, Bangkok, 2001, p. 359.

3 T. Chaowasku and P. J. A. Keßler, Nord. J. Bot., 2013, 3, 680699.

4 G. G. Harrigan, A. A. L. Gunatilaka, D. G. I. Kingston, G. W. Chan and R. K. Johnson, J. Nat. Prod., 1994, 57, 68-73.

5 R. Wu, Q. Ye, N. Y. Chen and G. L. Zhang, Chin. Chem. Lett., 2001, 12, 247-248.

6 C. Kamperdick, N. H. Van and T. V. Sung, Phytochemistry, 2002, 61, 991-994.

7 D. T. Huong, C. Kamperdick and T. V. Sung, J. Nat. Prod., 2004, 67, 445-447.

8 Y. Lei, L. Wu, H. Shi and P. Tu, Helv. Chim. Acta, 2008, 91, 495-500.

9 N. P. Thao, B. T. T. Luyen, B. H. Tai, N. M. Cuong, Y. C. Kim, C. V. Minh and Y. H. Kim, Bioorg. Med. Chem. Lett., 2015, 25, 3859-3863.

10 K. Sawasdee, T. Chaowasku and K. Likhitwitayawuid, Molecules, 2010, 15, 639-648.

11 K. Sawasdee, T. Chaowasku, V. Lipipun, T. H. Dufat, S. Michel and K. Likhitwitayawuid, Fitoterapia, 2013, 85, 49-56.

12 K. Sawasdee, T. Chaowasku, V. Lipipun, T. H. Dufat, S. Michel and K. Likhitwitayawuid, Tetrahedron Lett., 2013, 54, 4259-4263.

13 K. Sawasdee, T. Chaowasku, V. Lipipun, T. H. Dufat, S. Michel, V. Jongbunprasert and K. Likhitwitayawuid, Biochem. Syst. Ecol., 2014, 54, 179-181.

14 B. Chen, C. Feng, B. Li and G. Zhang, Nat. Prod. Res., 2003, 17, 397-402.

15 H. Zhang, C. Ma, N. V. Hung, N. M. Cuong, G. T. Tan, B. D. Santarsiero, A. D. Mesecar, D. D. Soejarto, J. M. Pezzuto and H. H. S. Fong, J. Med. Chem., 2006, 49, 693-708.

16 C. Naphong, W. Pompimon and P. Sombutsiri, Am. J. Appl. Sci., 2013, 10, 787-792.

17 S. Jumana, C. M. Hasan and M. A. Rashid, Fitoterapia, 2000, 71, 559-561.

18 C. M. Hasan, S. Jumana and M. A. Rashid, Nat. Prod. Lett., 2000, 14, 393-397.

19 S. Jumana, C. M. Hasan and M. A. Rashid, Biochem. Syst. Ecol., 2000, 28, 483-485.

20 N. Wongsa, S. Kanokmedhakul and K. Kanokmedhakul, Phytochemistry, 2011, 72, 1859-1864.

21 N. Wongsa, S. Kanokmedhakul and K. Kanokmedhakul, Phytochemistry, 2015, 109, 154.

22 I. Ohtani, T. Kusumi, Y. Kashman and H. Kakisawa, J. Am. Chem. Soc., 1991, 113, 4092-4096.

23 Bruker, APEX2, SAINT and SADABS, Bruker AXS Inc., Madison, WI, USA, 2014.

24 G. M. Sheldrick, Acta Crystallogr., Sect. A: Found. Crystallogr., 2008, A64, 112. 
25 W. Trager and J. B. Jensen, Science, 1967, 193, 673-675.

26 R. E. Desjardins, C. J. Canfield, J. D. Haynes and J. D. Chulay, Antimicrob. Agents Chemother., 1979, 16, 710-718.

27 D. A. Scudiere, R. H. Shoemaker, K. D. Paull, A. Monks, S. Tierney, T. H. Nofziger, M. J. Currens, D. Seniff and M. R. Boyd, Cancer Res., 1988, 48, 4827-4833.

28 J. O'Brien, I. Wilson, T. Orton and F. Pognan, Eur. J. Biochem., 2000, 267, 5421-5426.

29 L. Hunt, M. Jordan, M. D. Jesus and F. M. Wurm, Biotechnol. Bioeng., 1999, 65, 201-205.

30 T. Hashimoto, M. Suganuma, H. Fujiki, M. Yamada, T. Kohno and Y. Asakawa, Phytomedicine, 2003, 10, 309-317.

31 Y. Ogawa, H. Oku, E. Iwaoka, M. Iinuma and K. Ishiguro, Chem. Pharm. Bull., 2007, 54, 675-678.

32 J. M. J. Vasconcelos, A. M. S. Silva and J. A. S. Cavaleiro, Phytochemistry, 1998, 49, 1421-1424.

33 E. Lee, B. H. Moon, Y. Park, S. Hong, S. Lee, Y. Lee and Y. Lim, Bull. Korean Chem. Soc., 2008, 29, 507-510.
34 J. T. B. Ferreira, R. C. Boscaini and F. A. Marques, Nat. Prod. Lett., 1993, 2, 313-316.

35 A. G. Ferreira, J. B. Fernandes, P. C. Vieira, O. R. Gottlieb and H. E. Gottlieb, Phytochemistry, 1995, 40, 1723-1728.

36 A. G. Ferreira, M. Motidome, O. R. Gottlieb, J. B. Fernandes, P. C. Vieira, M. Cojocaru and H. E. Gottlieb, Phytochemistry, 1989, 28, 579-583.

37 A. G. Rücker, E. Schenkel, D. Manns, M. Falkenberg and A. Marek, Phytochemistry, 1996, 41, 297-300.

38 Y.-S. Wang, R. Li, Y. Huang, W.-B. Shang, F. Chen, H.-B. Zhang and J.-H. Yang, Phytochem. Lett., 2013, 6, 26-30.

39 T. Sagawa, Y. Takaishi, Y. Fujimoto, C. Duque, C. Osorio, F. Ramos, C. Garzon, M. Sata, M. Okamoto, T. Oshikawa and S. U. Ahmed, J. Nat. Prod., 2005, 68, 502-505.

40 M. Fujiki, Symmetry, 2014, 6, 677-703.

41 http://www.nmr.ch.tum.de/home/dames/J_reich_uwisc.pdf, July 2015. 